\title{
Degenerate scale problem when solving Laplace's equation by BEM and its treatment
}

\author{
J. T. Chen ${ }^{*} \dagger, 1$, S. R. $\operatorname{Lin}^{1}$ and K. H. Chen ${ }^{2}$ \\ ${ }^{1}$ Department of Harbor and River Engineering, National Taiwan Ocean University, Keelung, Taiwan \\ ${ }^{2}$ Department of Information Management, Toko University, Chia-Yi, Taiwan
}

\begin{abstract}
SUMMARY
In this paper, Laplace problems are solved by using the dual boundary element method (BEM). It is found that a degenerate scale problem occurs if the conventional BEM is used. In this case, the influence matrix is rank deficient and numerical results become unstable. Both the circular and elliptical bars are studied analytically in the continuous system. In the discrete system, the Fredholm alternative theorem in conjunction with the SVD (Singular Value Decomposition) updating documents is employed to sort out the spurious mode which causes the numerical instability. Three regularization techniques, method of adding a rigid body mode, hypersingular formulation and CHEEF (Combined Helmholtz Exterior integral Equation Formulation) concept, are employed to deal with the rankdeficiency problem. The addition of a rigid body term, $c$, in the fundamental solution is proved to shift the original degenerate scale to a new degenerate scale by a factor $\mathrm{e}^{-c}$. The torsion rigidities are obtained and compared with analytical solutions. Numerical examples including elliptical, square and triangular bars were demonstrated to show the failure of conventional BEM in case of the degenerate scale. After employing the three regularization techniques, the accuracy of the proposed approaches is achieved. Copyright (c) 2004 John Wiley \& Sons, Ltd.
\end{abstract}

KEY WORDS: boundary element method; degenerate scale; degenerate kernel; hypersingular formulation; CHEEF concept; Fredholm alternative theorem; SVD updating document

\section{INTRODUCTION}

During the last three decades, boundary element method (BEM) has been recognized as an acceptable tool for engineering analysis $[1,2]$. However, there still exists some pitfalls imbedded in the BEM, e.g. rank-deficiency problems. The well-known one is the fictitious (irregular) frequency in the exterior acoustics. Burton and Miller [3] solved the problem by combining singular and hypersingular equations with an imaginary constant. Chen et al. [4]

\footnotetext{
*Correspondence to: J. T. Chen, Department of Harbor and River Engineering, National Taiwan Ocean University, Keelung, Taiwan.

†E-mail: jtchen@mail.ntou.edu.tw

Contract/grant sponsor: National Science Council; contract/grant number: NSC-90-2211-E-019-021
}

Received 6 August 2002

Copyright (c) 2004 John Wiley \& Sons, Ltd.

Revised 26 March 2004

Accepted 7 May 2004 
extended the Burton and Miller method to filter out the spurious eigenvalues in the multiply connected eigenproblem. Schenck [5] proposed a Combined Helmholtz Interior integral Equation Formulation (CHIEF) method, which is easy to implement by applying the integral equation on a number of points located outside the domain of interest. It is efficient to overcome the problem of non-unique solutions in case of fictitious frequency, but it still has some drawbacks since the chosen point may fail. How to determine the number of points and how to choose their positions were discussed by Chen et al. [6]. In a similar way for the interior eigenproblem, the CHEEF technique [7] instead of the CHIEF concept was applied to filter out spurious eigenvalues successfully by adding constraints from the points outside the domain in the multiple reciprocity BEM [8], real-part BEM [9] and imaginary-part BEM [10]. Rankdeficiency problems also occur when BEM is applied to deal with crack or corner problems. Dual formulation in conjunction with the hypersingular equation has received much attention in the last decade. A review article can be found in Reference [11].

In the BEM implementation, the rigid body motion or the so-called constant potential test is always employed to examine the singular matrices of strongly singular kernels and hypersingular kernels for the problems without degenerate boundaries. Lutz et al. [12] termed it a simple solution. Based on this concept, diagonal terms of a singular influence matrix can be easily determined. Singular matrix occurs physically and mathematically in the sense that the nonunique solution for the singular matrix implies an arbitrary rigid body term for the interior Neumann (traction) problem. However, the influence matrix of the weakly singular kernel may be singular for the Dirichlet problem [13] when the geometry is special. The non-unique solution is not physically realizable but results from the zero singular value in the influence matrix by using the BEM. From the point of view of linear algebra, the problem also originates from the rank deficiency in the influence matrices. For example, the non-unique solution of a circle with a unit radius has been noted by Petrovsky [14] and by Jaswon and Symm [15]. The special geometry which results in a non-unique solution for a potential problem is called 'degenerate scale'. The term 'scale' stems from the fact that the numerical instability of a unit circle of radius $1 \mathrm{~m}(1 \mathrm{~cm})$ disappears if the radius of $100 \mathrm{~cm}(0.01 \mathrm{~m})$ is used in the BEM implementation. Christiansen [16,17] termed it a critical value (C.V.) since it is mathematically realizable. In real implementation, we need to avoid the number one for the circular radius using the normalized scale. The numerical difficulties due to non-uniqueness of solutions have been solved by using the necessary and sufficient boundary integral equation (NSBIE) [18-21] and boundary contour method [22]. Also, the degenerate scale of multiply connected problems was discussed for the Laplace equation by Tomlinson et al. [23]. The non-unique solution in biharmonic problems was also studied by Mitra and Das [24] and Christiansen [16]. Chen et al. $[25,26]$ studied the degenerate scale for the simply and multiply connected problems by using the degenerate kernels and circulants in a discrete system for circular and annular cases. Mathematically speaking, the singularity pattern distributed along a ring boundary resulting in a null-field interior solution introduces a degenerate scale. This concept was also extended to study the spurious eigenvalues for annular cavities by Chen et al. [4]. The similar application to the two-dimensional elasticity was addressed in Reference [27]. A rigorous study was proposed mathematically by Kuhn [28] and Constanda [29,30] for the occurring mechanism of the degenerate scale. SVD technique has been used to detect the non-unique solution in case of degenerate scale $[17,31]$. A complete collection of integral equations and functional equations for solving torsion problem has been written down in Reference [32]. However, very few numerical results to deal with the degenerate scale or critical value have been worked out. Two 
methods to eliminate degenerate scale, scaling method and restriction method were discussed by Christiansen [33]. He also investigated the condition number of the influence matrix of the fictitious BEM and null-field approach [34]. Here, three regularization techniques will be employed to avoid the zero singular value. One alternative to treat the problem is to superimpose a rigid body term in the fundamental solution for the BEM formulation. Although the original degenerate scale problem can be circumvented for the special geometry, the new degenerate scale will be proved to move to another size. Another alternative of hypersingular formulation is employed to avoid the zero eigenvalue in paying the price of determining the Hadamard principal value. By adopting the CHEEF concept for obtaining an independent constraint, we can also deal with the degenerate scale problems free of hypersingularity.

In this paper, we will focus on the analytical investigation for the phenomenon of degenerate scales in the BEM for torsion problems in continuous and discrete systems. The degenerate scale for the elliptical bar under torsion will be derived analytically in a continuous system by using the elliptical co-ordinate. Circular domain is a special case for check. The degenerate kernel and circulant will be employed to derive the degenerate scale in the continuous and discrete systems, respectively. Also, the addition of rigid body term $c$ in the fundamental solution will be proved to move the original degenerate scale to the new degenerate scale by a factor of $\mathrm{e}^{-c}$. In the discrete system, the Fredholm alternative theorem in conjunction with SVD updating document will be employed to find the degenerate scale and the corresponding spurious mode. The relation between the spurious mode and unitary vector in SVD will be constructed. Also, we will propose three alternatives, method of adding a rigid body mode, hypersingular formulation and CHEEF technique, to overcome the non-unique solution in the numerical implementation. Method of adding a rigid body mode in the fundamental solution can shift the zero singular value in the conventional BEM. Instead of using the conventional BEM, the second equation in the dual BEM, i.e. hypersingular formulation, can avoid the zero singular value. By using the CHEEF technique, the addition of a constraint by collocating the points outside the domain can promote the rank of the singular matrix. Numerical examples, torsion problems of elliptical, square, triangular bars, will be demonstrated to see the numerical instability for the degenerate scale problems. The treatment for the suppression of numerical instability will be done.

\section{DUAL BOUNDARY INTEGRAL FORMULATION AND DUAL BEM FOR TORSION PROBLEMS}

In mathematical physics, many engineering problems can be described by the Laplace equation. For simplicity, torsion problem is considered here. The torsion problem of a bar with an arbitrary cross section in Figure 1 can be formulated by the Poisson equation as follows [35, 36]:

$$
\nabla^{2} u^{*}\left(x_{1}, x_{2}\right)=-2, \quad\left(x_{1}, x_{2}\right) \in D
$$

where $u^{*}$ is the torsion (Prandtl) function, $\nabla^{2}$ is the Laplacian operator and $D$ is the domain. The boundary condition is

$$
u^{*}\left(x_{1}, x_{2}\right)=0, \quad\left(x_{1}, x_{2}\right) \in B
$$

where $B$ is the boundary. Since Equation (1) contains the body source term which results in a domain integral by using the BEM, the problem can be reformulated to

$$
\nabla^{2} u\left(x_{1}, x_{2}\right)=0, \quad\left(x_{1}, x_{2}\right) \in D
$$




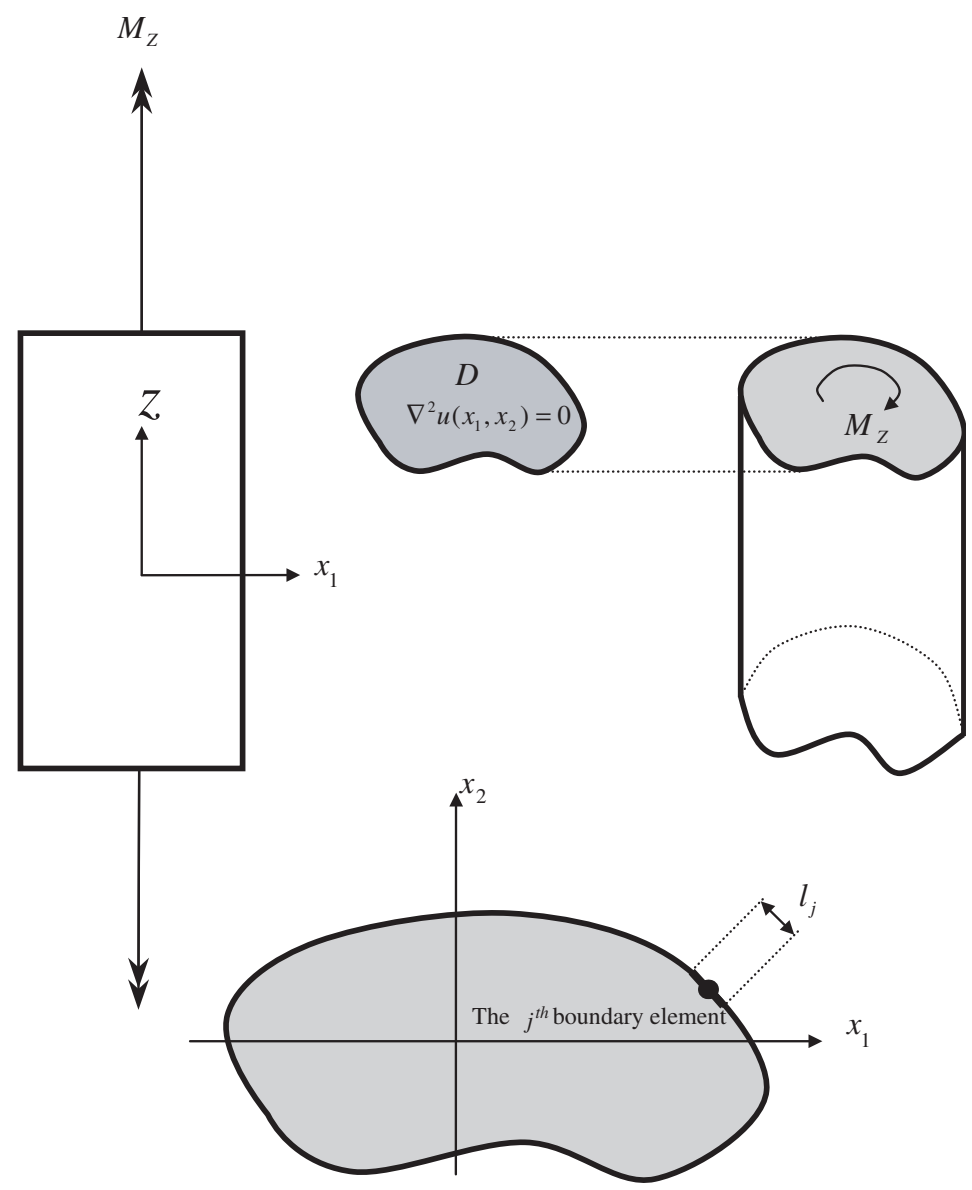

Figure 1. Figure sketch of the torsion problem.

and the boundary condition is changed to

$$
u\left(x_{1}, x_{2}\right)=\tilde{u}, \quad\left(x_{1}, x_{2}\right) \in B
$$

where the torsion function $u^{*}$ can be obtained from $u$ by superimposing $\tilde{u}, u=u^{*}+\tilde{u}$ and $\tilde{u}=\left(x_{1}^{2}+x_{2}^{2}\right) / 2$.

This new model for the torsion problem using Equation (3) is the Laplace equation subject to the Dirichlet data of Equation (4), which is very easy to implement using the DBEM, e.g. the BEPO2D program can be used in this study [37]. The torque, $M_{z}$, can then be determined by

$$
M_{z}=\iint_{D}\left(x_{1} \tau_{23}-x_{2} \tau_{13}\right) \mathrm{d} x_{1} \mathrm{~d} x_{2}
$$


where $\tau_{23}$ and $\tau_{13}$ are the shearing stresses determined by $\tau_{23}=-\kappa G \partial u^{*} / \partial x_{1}$ and $\tau_{13}=$ $\kappa G \partial u^{*} / \partial x_{2}$, in which $G$ is the shear modulus and $\kappa$ denotes the twist angle per unit length.

By employing the Green's second identity and Equation (1), the area integral in Equation (5) can be transformed into a boundary integral and a domain integral as follows [35]:

$$
\begin{aligned}
M_{z} & =\iint_{D}\left(x_{1} \tau_{23}-x_{2} \tau_{13}\right) \mathrm{d} x_{1} \mathrm{~d} x_{2} \\
& =-\kappa G \oint_{B} \tilde{u} \frac{\partial u^{*}}{\partial n} \mathrm{~d} B-\kappa G \iint_{D}\left(x_{1}^{2}+x_{2}^{2}\right) \mathrm{d} x_{1} \mathrm{~d} x_{2}
\end{aligned}
$$

The induced area integral of the second term on the right-hand side of the equal sign in Equation (6) can be reformulated into a boundary integral again by using the Gauss theorem as follows [35]:

$$
-\kappa G \iint_{D}\left(x_{1}^{2}+x_{2}^{2}\right) \mathrm{d} x_{1} \mathrm{~d} x_{2}=\frac{-\kappa G}{16} \oint_{B} \frac{\partial\left\{\left(x_{1}^{2}+x_{2}^{2}\right)^{2}\right\}}{\partial n} \mathrm{~d} B
$$

The torsion problem can be simulated by using the mathematical model of the Laplace equation as shown in Equation (3). Now, we will consider the boundary integral formulation for numerical analysis. Using the Green's identity, the first equation of the dual boundary regular integral equations for the domain point $x$ can be derived as follows:

$$
2 \pi u(x)=\int_{B} T(s, x) u(s) \mathrm{d} B(s)-\int_{B} U(s, x) \frac{\partial u(s)}{\partial n_{s}} \mathrm{~d} B(s)
$$

where

$$
\begin{aligned}
& U(s, x) \equiv \ln (r) \\
& T(s, x) \equiv \frac{\partial U(s, x)}{\partial n_{s}}
\end{aligned}
$$

in which $r$ is the distance between the field point $x$ and the source point $s$, and $n_{s}$ is the normal vector for the boundary point $s$. After taking the normal derivative of Equation (8), the second equation of the dual boundary regular integral equations for the domain point $x$ can be derived as

$$
2 \pi \frac{\partial u(x)}{\partial n_{x}}=\int_{B} M(s, x) u(s) \mathrm{d} B(s)-\int_{B} L(s, x) \frac{\partial u(s)}{\partial n_{s}} \mathrm{~d} B(s)
$$

where

$$
\begin{gathered}
L(s, x) \equiv \frac{\partial U(s, x)}{\partial n_{x}} \\
M(s, x) \equiv \frac{\partial^{2} U(s, x)}{\partial n_{x} \partial n_{s}}
\end{gathered}
$$


in which $n_{x}$ is the normal vector for the field point $x$. Equations (8) and (11) are coined the dual boundary regular integral equations for the domain point $x$. The explicit forms of the kernel functions can be found in Reference [37]. By tracing the field point $x$ to the boundary, the dual boundary singular integral equations for the boundary point $x$ can be derived as

$$
\begin{gathered}
\pi u(x)=\text { C.P.V. } \int_{B} T(s, x) u(s) \mathrm{d} B(s)-\text { R.P.V. } \int_{B} U(s, x) \frac{\partial u(s)}{\partial n_{s}} \mathrm{~d} B(s) \\
\pi \frac{\partial u(x)}{\partial n_{x}}=\text { H.P.V. } \int_{B} M(s, x) u(s) \mathrm{d} B(s)-\text { C.P.V. } \int_{B} L(s, x) \frac{\partial u(s)}{\partial n_{s}} \mathrm{~d} B(s)
\end{gathered}
$$

where R.P.V., C.P.V. and H.P.V. denote the Riemann principal value, Cauchy principal value and Hadamard or Mangler principal value, respectively. After discretizing the boundary into $2 N$ boundary elements, Equations (14) and (15) reduce to

$$
\begin{aligned}
& {[U]_{2 N \times 2 N}\{t\}_{2 N \times 1}=[T]_{2 N \times 2 N}\{u\}_{2 N \times 1}} \\
& {[L]_{2 N \times 2 N}\{t\}_{2 N \times 1}=[M]_{2 N \times 2 N}\{u\}_{2 N \times 1}}
\end{aligned}
$$

where $[U],[T],[L]$ and $[M]$ are the four influence matrices which can be found in Reference [37], $\{u\}$ and $\{t\}$ are the boundary data for the primary and the secondary boundary variables, respectively.

To determine the torsion rigidity using Equation (6), the following boundary integral can be integrated numerically as follows:

$$
\oint_{B} \tilde{u} \frac{\partial u^{*}}{\partial n} \mathrm{~d} B=\oint_{B} \tilde{u} \frac{\partial u}{\partial n} \mathrm{~d} B-\oint_{B} \tilde{u} \frac{\partial \tilde{u}}{\partial n} \mathrm{~d} B=\sum_{j=1}^{2 N} \tilde{u}_{j}\left[\left(\frac{\partial u}{\partial n}\right)_{j}-\left(\frac{\partial \tilde{u}}{\partial n}\right)_{j}\right] l_{j}
$$

where $(\partial u / \partial n)_{j}$ is the normal derivative of $u$ for the $j$ th boundary element, $l_{j}$ is the length of the $j$ th boundary element and another boundary integral in Equation (7) can be discretized as follows:

$$
\oint_{B} \frac{\partial\left\{\left(x_{1}^{2}+x_{2}^{2}\right)^{2}\right\}}{\partial n} \mathrm{~d} B=4 \sum_{j=1}^{2 N}\left(\frac{\partial \tilde{u}^{2}}{\partial n}\right)_{j} l_{j}
$$

\section{PROOF OF THE EXPANSION RATIO OF $\mathrm{e}^{-c}$ FOR THE NEW DEGENERATE SCALE AFTER ADDING A RIGID BODY TERM $c$ IN THE FUNDAMENTAL SOLUTION}

For a two-dimensional potential problem, there exists a unique solution for $\psi_{1}(s)$ satisfying

$$
u(x)=\int_{B} U(s, x) \psi_{1}(s) \mathrm{d} B(s), \quad x \in B
$$

where $B$ is the normal boundary with the enclosing domain $D$. For simplicity, we can assume a constant potential field since it is a 'simple solution' for the Laplace equation. Equation (20) 
reduces to

$$
1=\int_{B} U(s, x) \psi_{1}(s) \mathrm{d} B(s), \quad x \in B
$$

When the degenerate scale $B_{d}$ occurs, the non-unique solution of Equation (21) implies that

$$
0=\int_{B_{d}} U(s, x) \psi_{1}(s) \mathrm{d} B(s), \quad x \in B_{d}
$$

has a non-trivial solution for $\psi_{1}(s)$, where $B_{d}$ is the boundary of degenerate scale using the fundamental solution $U(s, x)=\ln (r)$ [13,15-17, 32-34].

For determining the degenerate scale systematically from one trial of a normal scale, we provide a flowchart as shown in Figure 2(a) and the numerical results as shown in Table I.

\section{Theorem}

The boundary of $g\left(x_{1}, x_{2}\right)=0$ shown in Figure 2(b), which is a degenerate scale using the fundamental solution $(U(s, x)=\ln (r))$ is changed to a new degenerate scale of $g\left(x_{1} / \mathrm{e}^{-c}, x_{2} / \mathrm{e}^{-c}\right)=0$ using the modified fundamental solution $\left(U^{*}(s, x)=\ln (r)+c\right)$.

Proof

If the degenerate scale $B_{d}\left(g\left(x_{1}, x_{2}\right)=0\right)$ occurs, the fundamental solution $U(s, x)$ can be modified to $U(s, x)+c$ to avoid the singular case. In other words, there is a unique solution $\psi_{1}(s)$ for the following equation:

$$
1=\int_{B_{d}}[U(s, x)+c] \psi_{1}(s) \mathrm{d} B(s)
$$

We expand the normal boundary $B_{d}(U(s, x)=\ln (r)+c)$ in Equation (23) to the "new degenerate scale', $B_{d^{*}}$, by using the modified fundamental solution as shown in Figure 2 (a). The homogeneous Equation (23) reduces to

$$
0=\int_{B_{d^{*}}}\left[U\left(s d^{*}, x d^{*}\right)+c\right] \psi_{1}\left(s d^{*}\right) \mathrm{d} B\left(s d^{*}\right)
$$

where $d^{*}$ is the expansion ratio. In the new degenerate scale, $B_{d^{*}}$, for the case of modified fundamental solution $(U(s, x)=\ln (r)+c)$, it means that Equation (24) has a non-trivial solution. By using mapping properties, $\mathrm{d} B\left(s d^{*}\right)=d^{*} \mathrm{~d} B(s)$ and $U\left(s d^{*}, x d^{*}\right)=U(s, x)+\ln d^{*}$, Equation (24) reduces to

$$
\begin{aligned}
0 & =d^{*} \int_{B_{d}}\left[\left(U(s, x)+\ln \left(d^{*}\right)+c\right] \psi_{1}(s) \mathrm{d} B(s)\right. \\
& =d^{*} \ln \left(d^{*}\right) \int_{B_{d}} \psi_{1}(s) \mathrm{d} B(s)+c d^{*} \int_{B_{d}} \psi_{1}(s) \mathrm{d} B(s)+d^{*} \int_{B_{d}} U(s, x) \psi_{1}(s) \mathrm{d} B(s)
\end{aligned}
$$


(a)

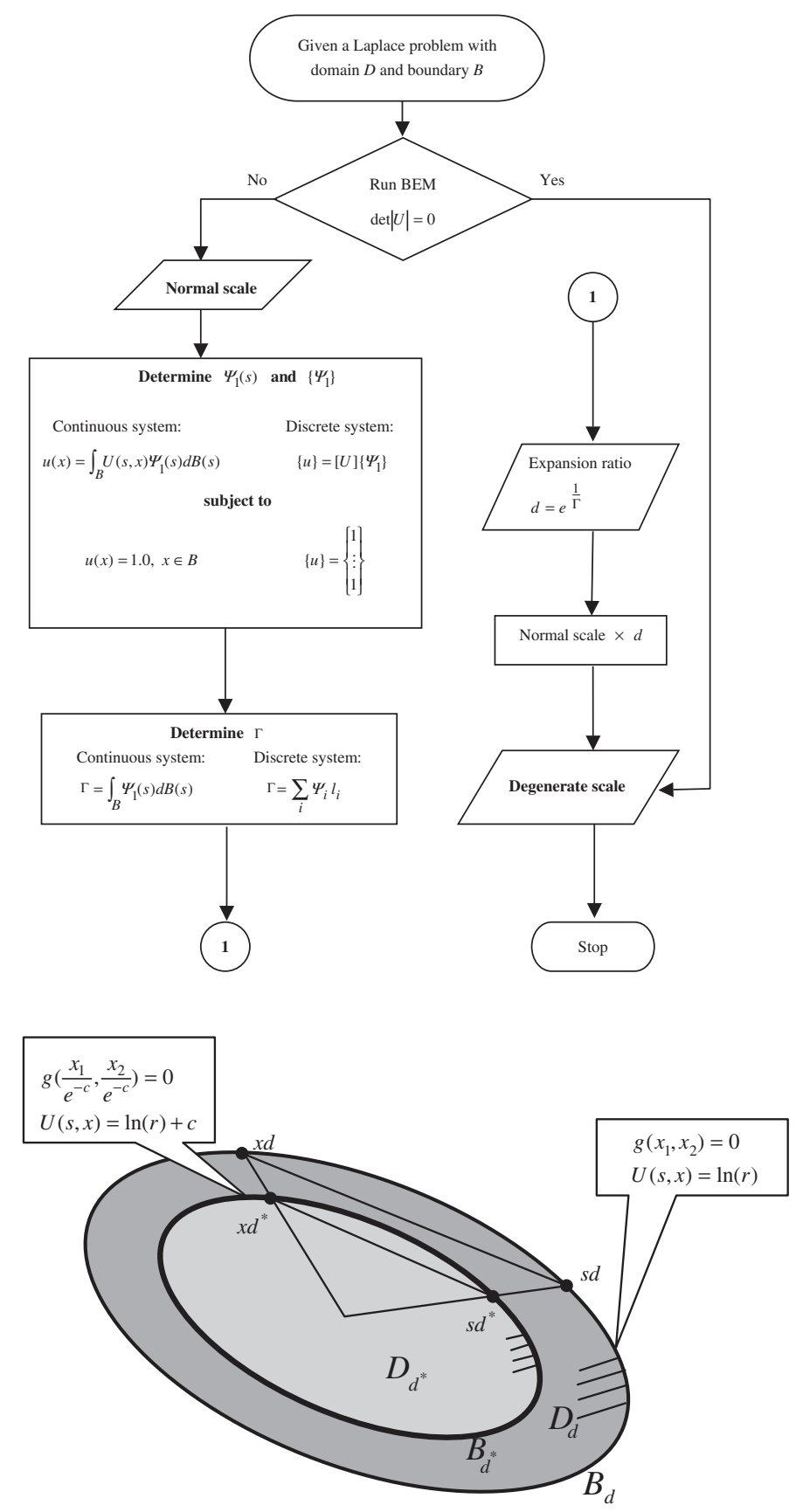

Figure 2. (a) The flowchart of determining the degenerate scale systematically; and (b) The original degenerate scale domain $D_{d}$ using $U(s, x)=\ln (r)$ and the modified degenerate scale $D_{d^{*}}$ using $U(s, x)=\ln (r)+c$. 


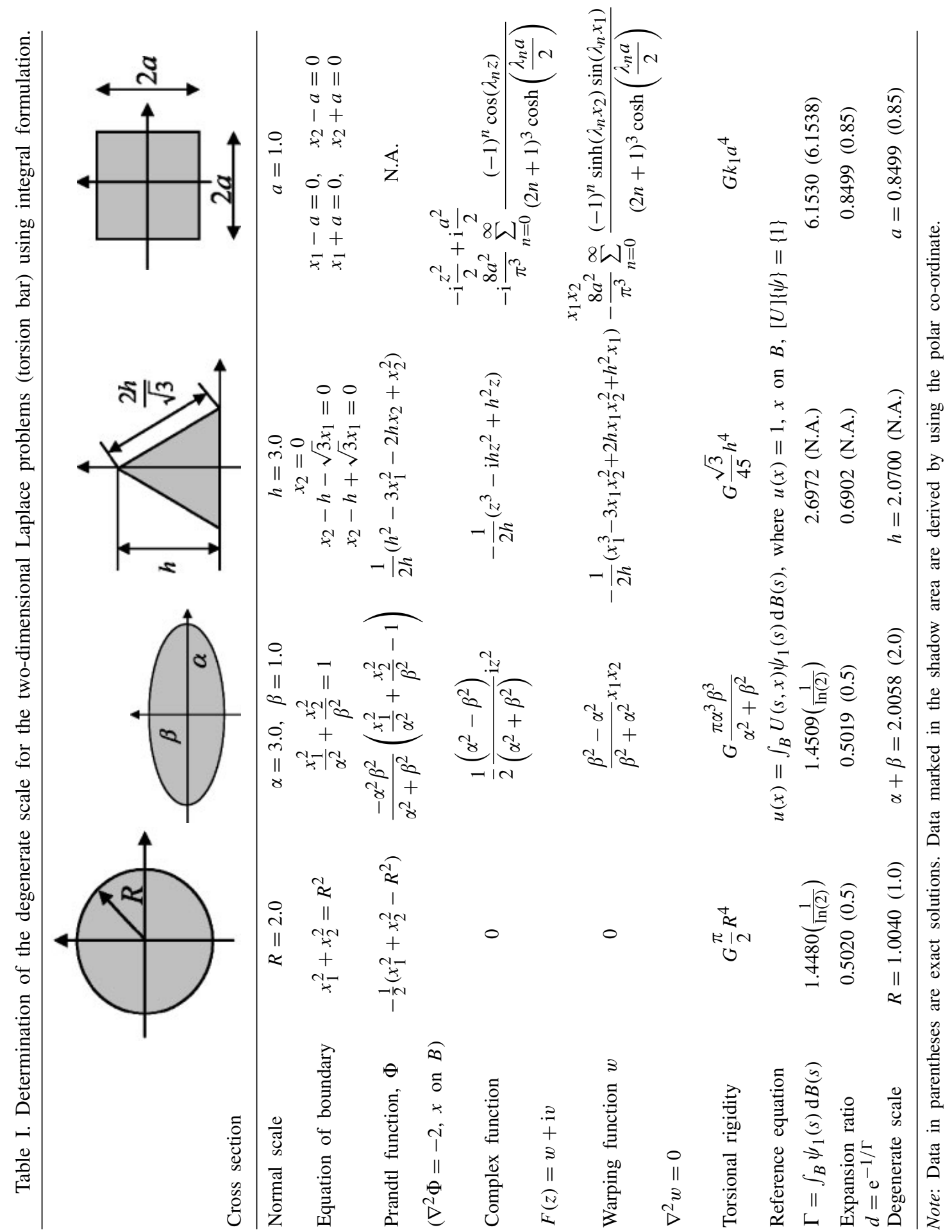

Copyright (c) 2004 John Wiley \& Sons, Ltd. 
Since $\int_{B_{d}} U(s, x) \psi_{1}(s) \mathrm{d} B(s)=0$ in the original degenerate scale, Equation (25) simplifies to

$$
\begin{aligned}
0 & =d^{*} \ln \left(d^{*}\right) \int_{B_{d}} \psi_{1}(s) \mathrm{d} B(s)+c d^{*} \int_{B_{d}} \psi_{1}(s) \mathrm{d} B(s) \\
& =\left(\ln \left(d^{*}\right)+c\right) \int_{B_{d}} \psi_{1}(s) \mathrm{d} B(s)
\end{aligned}
$$

The expansion ratio, $d^{*}$, satisfying

$$
d^{*}=\mathrm{e}^{-c}
$$

results in a new degenerate scale in Equation (27). To demonstrate the accuracy of Equation (27), a special case of circular bar will be discussed in the following section in detail.

\section{MATHEMATICAL ANALYSIS OF THE DEGENERATE SCALE FOR AN ELLIPTICAL BAR UNDER TORSION}

For an elliptical bar under torsion as shown in Figure 3(a), the governing equation is also

$$
\nabla^{2} u\left(x_{1}, x_{2}\right)=0, \quad\left(x_{1}, x_{2}\right) \in D
$$

To study the degenerate scale for an elliptical bar [38], we consider an infinite domain and use the elliptic co-ordinate $\xi$ and $\eta$ defined by

$$
z=k \cosh \zeta, \quad \zeta=\xi+\mathrm{i} \eta
$$

where $z$ is the complex plane $\left(x_{1}+\mathrm{i} x_{2}\right), k$ is a constant and

$$
x_{1}=k \cosh \xi \cos \eta, \quad x_{2}=k \sinh \xi \sin \eta
$$

The co-ordinate $\xi$ is a constant and is equal to $\xi_{0}$ on the ellipse of the semiaxes $k \cosh \xi_{0}$ and $k \sinh \xi_{0}$ as shown in Figure 3(b). If the semiaxes are given as $\alpha$ and $\beta, k$ and $\xi_{0}$ can be determined by

$$
k=\sqrt{\alpha^{2}-\beta^{2}}, \quad \xi_{0}=\tanh ^{-1}\left(\frac{\alpha}{\beta}\right)
$$

We assume $u_{\mathrm{i}}$ and $u_{\mathrm{e}}$, for the interior and exterior potentials as shown in Figure 3(b), respectively,

$$
\begin{aligned}
& u_{\mathrm{i}}(\xi, \eta)=c_{1} \\
& u_{\mathrm{e}}(\xi, \eta)=c_{2}+c_{3} \xi
\end{aligned}
$$


(a)
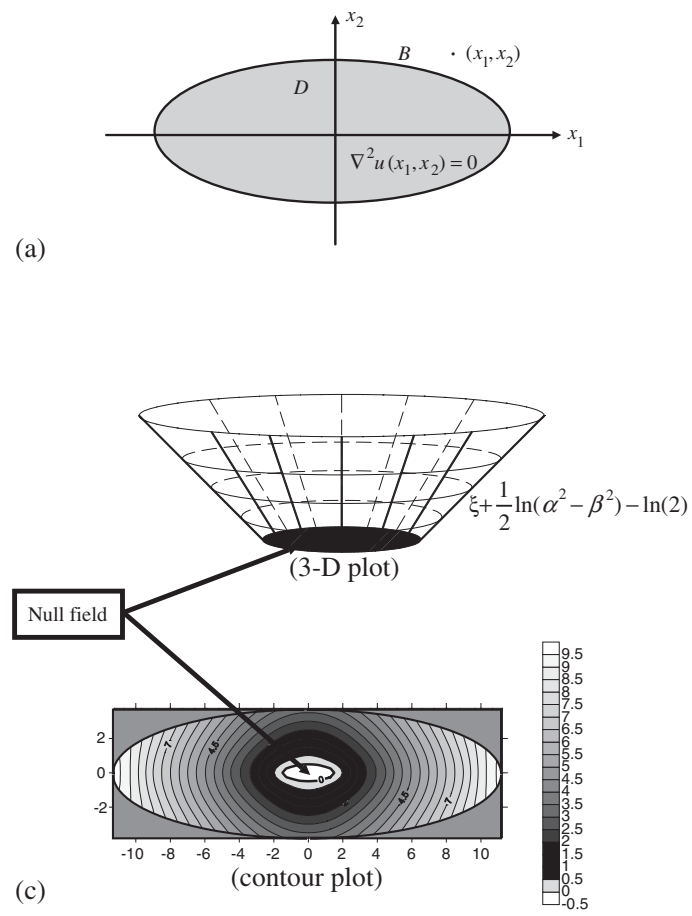

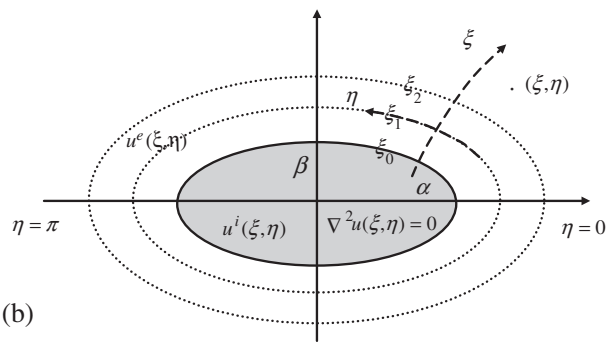

(b)

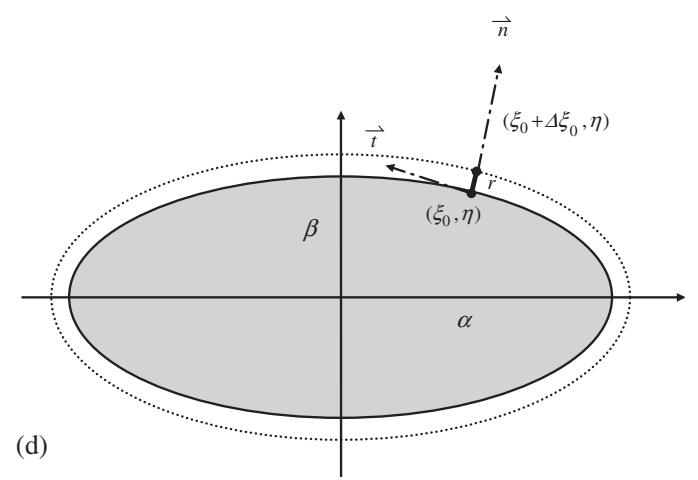

Figure 3. (a) Elliptical domain; (b) elliptical co-ordinate; (c) the null field for the elliptical domain; and (d) the positions of the points $\left(\xi_{0}, \eta\right)$ and $\left(\xi_{0}+\Delta \xi_{0}, \eta\right)$ in the elliptical co-ordinate.

where $c_{1}, c_{2}$ and $c_{3}$ are coefficients, and the subscripts ' $\mathrm{i}$ ' and 'e' denote the interior or exterior point separated by the elliptical boundary $\xi=\xi_{0}$, respectively. When $\xi$ approaches infinity, we have the asymptotic form

$$
r=|z|=|k \cosh \zeta| \simeq \frac{k}{2} \mathrm{e}^{\xi}
$$

and

$$
\xi \simeq \ln (r)-\ln \left(\frac{k}{2}\right)
$$

The exterior potential approaches $\ln r$ and the coefficient $c_{2}$ must be chosen as $c_{3} \ln (k / 2)$. The potential in the exterior domain is

$$
u_{\mathrm{e}}(\xi, \eta)=c_{3}\left(\xi+\ln \left(\frac{k}{2}\right)\right)
$$


On the other hand, when $\xi$ approaches $\xi_{0}$ on the elliptical boundary, we have

$$
\begin{aligned}
u_{\mathrm{e}}(\xi, \eta) & =c_{3}\left(\xi_{0}+\ln \left(\frac{k}{2}\right)\right) \\
& =c_{3}\left(\tanh ^{-1}\left(\frac{\beta}{\alpha}\right)+\frac{1}{2} \ln \left(\alpha^{2}-\beta^{2}\right)-\ln (2)\right)
\end{aligned}
$$

after using Equation (31). If we set

$$
\tanh (x)=\left(\frac{\mathrm{e}^{2 x}-1}{\mathrm{e}^{2 x}+1}\right)=\chi
$$

we have

$$
x=\frac{1}{2} \ln \left(\frac{1+\chi}{1-\chi}\right)=\tanh ^{-1} \chi
$$

By setting $\chi$ to be $(\beta / \alpha)$, we have

$$
\tanh ^{-1}\left(\frac{\beta}{\alpha}\right)=\frac{1}{2} \ln \left(\frac{\alpha+\beta}{\alpha-\beta}\right)
$$

The exterior potential in Equation (37) becomes

$$
\begin{aligned}
u_{\mathrm{e}}(\xi, \eta) & =c_{3}\left(\frac{1}{2} \ln \left(\frac{\alpha+\beta}{\alpha-\beta}\right)+\frac{1}{2} \ln \left(\alpha^{2}-\beta^{2}\right)-\ln (2)\right) \\
& =c_{3} \ln \left(\frac{\alpha+\beta}{2}\right)
\end{aligned}
$$

For the continuity of displacement across the boundary, the displacement by approaching from the exterior domain must equal to the potential by approaching from the interior domain. We have

$$
c_{1}=\ln \left(\frac{\alpha+\beta}{2}\right) c_{3}
$$

and the potential can be written as

$$
\begin{aligned}
& u_{\mathrm{i}}(\xi, \eta)=c_{3} \ln \left(\frac{\alpha+\beta}{2}\right) \\
& u_{\mathrm{e}}(\xi, \eta)=c_{3}\left[\xi+\frac{1}{2} \ln \left(\alpha^{2}-\beta^{2}\right)-\ln (2)\right]
\end{aligned}
$$

The degenerate scale occurs for the interior null field when the relationship between $\alpha$ and $\beta$ is $\alpha+\beta=2$, i.e. $c_{1}=0$. In such a case, the strength of the singularity along the elliptical boundary cannot be determined in BEM implementation. This is the reason why a degenerate 
scale occurs. The fields for $u_{\mathrm{i}}$ and $u_{\mathrm{e}}$ are shown in Figure 3(c) for contour and three-dimensional plots. It is found that the null field is obtained in the ellipse. From Equations (30) and (31), the tangent vector $\tilde{\mathbf{t}}$ and normal vector $\tilde{\mathbf{n}}$ in Figure 3(d) can be derived as follows:

$$
\begin{aligned}
\tilde{\mathbf{t}} & =\left(-k \cosh \xi_{0} \sin \eta, \quad k \sinh \xi_{0} \cos \eta\right) \\
\tilde{\mathbf{n}} & =\left(k \sinh \xi_{0} \cos \eta, \quad k \cosh \xi_{0} \sin \eta\right)
\end{aligned}
$$

The exact solution for the normal flux on the boundary is

$$
\begin{aligned}
\frac{\partial u\left(\xi_{0}, \eta\right)}{\partial \tilde{\mathbf{n}}} & =\psi_{1}(\eta)=\frac{u\left(\xi_{0}+\Delta \xi_{0}, \eta\right)-u\left(\xi_{0}, \eta\right)}{r\left(\xi_{0}, \eta ; \xi_{0}+\Delta \xi_{0}, \eta\right)} \\
& =\frac{1}{\sqrt{\beta^{2} \cos ^{2} \eta+\alpha^{2} \sin ^{2} \eta}}
\end{aligned}
$$

where $r\left(\xi_{0}, \eta ; \xi_{0}+\Delta \xi_{0}, \eta\right)$ is the distance between the two points $\left(\xi_{0}, \eta\right)$ and $\left(\xi_{0}+\Delta \xi_{0}, \eta\right)$ in the elliptical co-ordinate, as shown in Figure 3(d). When $\alpha$ approaches $\beta$, the elliptical boundary becomes a circle and the degenerate scale is found to be $\alpha=\beta=1$. The result is the same in comparison with the degenerate scale in $[14,15]$. Equation $(47)$ reduces to $\psi_{1}(\eta)=1$ for the circle.

\section{SPECIAL CASE-A CIRCULAR BAR WITH A RADIUS $R$}

When $\alpha$ equals to $\beta$ in the elliptical case, it becomes a circular bar. The null field of Figure 3(c) is simplified to Figure 4 where $u^{\mathrm{e}}(\xi, \eta)=\ln r$ can be obtained from Equation (37) by setting $\alpha=\beta=R$. The degenerate scale occurs at the radius of one. In this case, $\phi(\eta)=\psi(\eta)=1$. For the discrete system of $2 N$ boundary elements, the influence matrix of $[U]$ is a symmetric circulant which can be decomposed by using SVD technique as

$$
[U]=[\Phi][\Sigma][\Psi]^{\mathrm{T}}
$$

where the singular values in the $[\Sigma]$ matrix are

$$
\sigma_{n}= \begin{cases}2 \pi R \ln (R), & n=0 \\ -\pi \frac{R}{|n|}, & n= \pm 1, \pm 2, \ldots, \pm(N-1), N\end{cases}
$$

After adding a rigid body term, $c$, in the fundamental solution, the influence matrix $[U]$ is modified to

$$
\left[U^{r}\right]=[U]+c^{*}\left\{\phi_{1}\right\}\left\{\psi_{1}\right\}^{\mathrm{T}}
$$




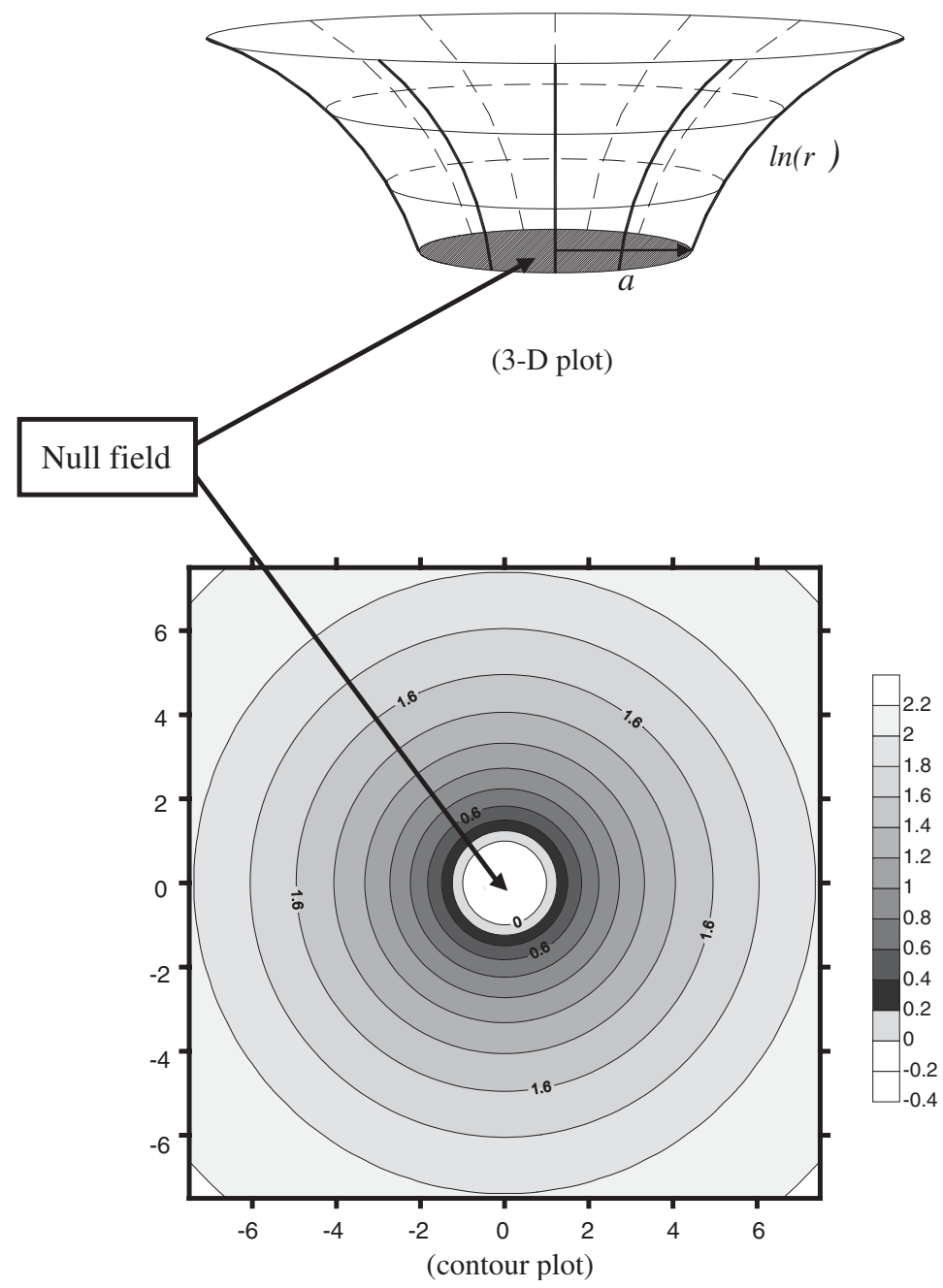

Figure 4. The null field for the circular domain.

where uniform mesh results in

$$
\left\{\phi_{1}\right\}=\left\{\psi_{1}\right\}=\frac{1}{\sqrt{2 N}}\left\{\begin{array}{c}
1 \\
1 \\
\vdots \\
1 \\
1
\end{array}\right\}_{2 N \times 1}
$$


We can easily obtain

$$
c^{*}=2 c N l=2 \pi r^{*} c
$$

In order to demonstrate that the rigid body term $c$ can shift the degenerate scale $R=1$ to another place $R=r^{*}$, the minimum singular value of the influence matrix $\left[U^{r}\right]$ becomes zero,

$$
2 \pi r^{*} \ln \left(r^{*}\right)+c^{*}=0
$$

Using Equation (52), we have

$$
2 \pi r^{*} \ln \left(r^{*}\right)+c 2 \pi r^{*}=0
$$

Equation (54) yields

$$
r^{*}=\mathrm{e}^{-c}
$$

Equation (27) is obtained again using the BEM. In the same way, we can prove Equation (27) in the continuous system. First, we define a boundary integral operator $\mathscr{U}$ which maps one boundary density function $p(s)$ to another boundary density function $q(x)$ as

$$
\mathscr{U}(p(s))=\lambda q(x)
$$

where the boundary integral operator, $\mathscr{U}$, is defined as

$$
\mathscr{U}(\psi(s))=\int_{B} U(s, x) \psi(s) \mathrm{d} B(s), \quad x \in B
$$

In this case, the associated eigenfunction for the zero eigenvalue is $\psi(s)=1$, i.e.

$$
\mathscr{U}(\psi(s))=\lambda \psi(x)=\int_{B} U(s, x) \psi(s) \mathrm{d} B(s)=0, \quad x \in B
$$

When the degenerate scale occurs, the eigenvalue, $\lambda$, is zero. By using the degenerate kernel function for the fundamental solution added by a rigid body term, $c$, [37], we have

$$
\begin{aligned}
U^{*}(s, x) & =U^{*}(R, \theta ; \rho, \phi) \\
& =\ln R-\sum_{m=1}^{\infty} \frac{1}{m}\left(\frac{\rho}{R}\right)^{m} \cos (m(\theta-\phi))+c
\end{aligned}
$$

where $x=(\rho, \phi)$ and $s=(R, \theta)$. For the circular case of radius one, the zero singular value results in a degenerate scale. After adding a rigid body term, $c$, the minimum singular value shifts to

$$
\sigma_{1}^{*}=2 \pi R \ln (R)+2 \pi R c
$$

We can obtain the radius with a unit length (free of rigid body term) is shifted to $\mathrm{e}^{-c}$ (after adding a rigid body term $c$ ) for keeping the zero singular value, see Figure 5 . In order to demonstrate that the rigid body term $c$ can shift the degenerate scale $(R=\rho=1)$ to another place $\left(R=r^{*}\right)$. Equation (61) can be rewritten as

$$
2 \pi r^{*} \ln \left(r^{*}\right)+2 \pi r^{*} c=0
$$




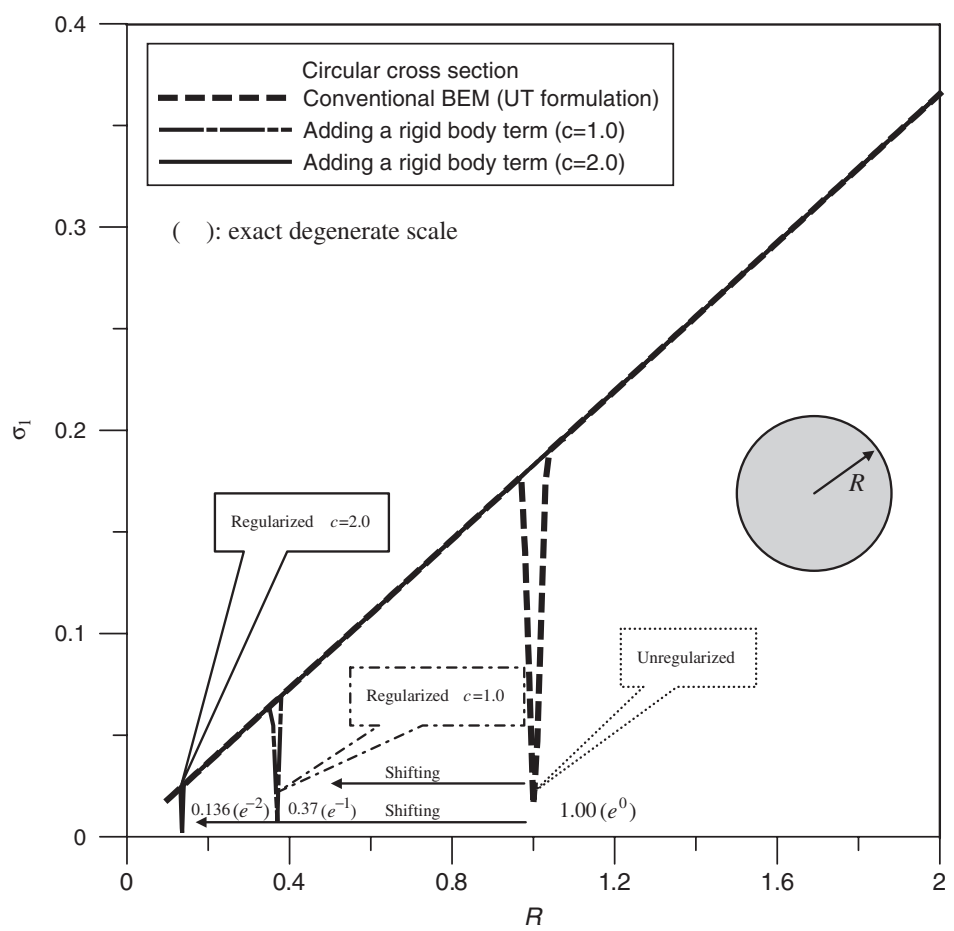

Figure 5. The first minimum singular value versus scale by using the conventional BEM (UT formulation) and adding a rigid body term for the circular bar.

Equation (62) yields

$$
r^{*}=\mathrm{e}^{-c}
$$

\section{DETECTION OF DEGENERATE SCALES AND DETERMINATION OF SPURIOUS MODES BY USING THE SVD UPDATING DOCUMENTS AND THE FREDHOLM ALTERNATIVE THEOREM}

\subsection{Fredholm alternative theorem}

The linear algebraic equation $[K]\{u\}=\{\bar{b}\}$ has a unique solution if and only if the only continuous solution to the homogeneous equation

$$
[K]\{u\}=\{0\}
$$

is $\{u\} \equiv\{0\}$. Alternatively, the homogeneous equation has at least one solution if the homogeneous adjoint equation

$$
[K]^{\mathrm{H}}\{\phi\}=\{0\}
$$


has a non-trivial solution $\{\phi\}$, where $[K]^{\mathrm{H}}$ is the transpose conjugate matrix of $[K]$ and $\{\bar{b}\}$ must satisfy the constraint $\left(\{\bar{b}\}^{\mathrm{H}}\{\phi\}=0\right)$. If the matrix $[K]$ is real, the transpose conjugate of a matrix is equal to its transpose only [39], i.e. $[K]^{\mathrm{H}}=[K]^{\mathrm{T}}$. By using the UT formulation, we have

$$
[U]\{t\}=[T]\{u\}=\{\bar{b}\}
$$

According to the Fredholm alternative theorem, Equation (66) has at least one solution for $\{t\}$ if the homogeneous adjoint equation

$$
[U]^{\mathrm{T}}\left\{\phi_{1}\right\}=\{0\}
$$

has a non-trivial solution $\left\{\phi_{1}\right\}$, in which the constraint $\left(\{\bar{b}\}^{\mathrm{T}}\left\{\phi_{1}\right\}=0\right)$ must be satisfied. By substituting $\bar{b}=[T]\{u\}$ in Equation (66) into $\{\bar{b}\}^{\mathrm{T}}\left\{\phi_{1}\right\}=0$, we obtain

$$
\{u\}^{\mathrm{T}}[T]^{\mathrm{T}}\left\{\phi_{1}\right\}=0
$$

Since $\{u\}$ is an arbitrary vector for the Dirichlet problem, we have

$$
[T]^{\mathrm{T}}\left\{\phi_{1}\right\}=\{0\}
$$

where $\left\{\phi_{1}\right\}$ is the spurious mode. Combining Equations (67) and (69) together, we have

$$
\left[\begin{array}{c}
{[U]^{\mathrm{T}}} \\
{[T]^{\mathrm{T}}}
\end{array}\right]\left\{\phi_{1}\right\}=\{0\} \quad \text { or } \quad\left\{\phi_{1}\right\}^{\mathrm{T}}[[U][T]]=\{0\}
$$

Equation (70) indicates that the two matrices have the same spurious mode $\left\{\phi_{1}\right\}$ corresponding to the same zero singular value when a degenerate scale occurs. The former one in Equation (70) is a form of updating term and the latter one is a form of updating document. By using the SVD technique for the $[U]^{\mathrm{T}}$ and $[T]^{\mathrm{T}}$ matrices, we have

$$
\begin{aligned}
& {[U]^{\mathrm{T}}=\left[\Psi_{U}\right]\left[\Sigma_{U}\right]\left[\Phi_{U}\right]^{\mathrm{T}}} \\
& {[T]^{\mathrm{T}}=\left[\Psi_{T}\right]\left[\Sigma_{T}\right]\left[\Phi_{T}\right]^{\mathrm{T}}}
\end{aligned}
$$

where $\left\{\phi_{1}\right\}$ is imbedded in both the matrices, $\left[\Phi_{U}\right]$ and $\left[\Phi_{T}\right]$, with the corresponding zero singular value in the matrices, $\left[\Sigma_{U}\right]$ and $\left[\Sigma_{T}\right]$, respectively. Since $\left\{\phi_{1}\right\}$ is one of the left unitary vector of $\left[\Phi_{U}\right]$ matrix with respect to the zero singular value, we have

$$
[U]^{\mathrm{T}}\left\{\phi_{1}\right\}=0\left\{\psi_{1}\right\}
$$

where $\left\{\phi_{1}\right\}$ and $\left\{\psi_{1}\right\}$ are the pair of non-trivial spurious modes which satisfy

$$
[U]\left\{\psi_{1}\right\}=0\left\{\phi_{1}\right\}
$$

The $\left\{\psi_{1}\right\}$ in Equation (73) for the discrete system and $\psi(\eta)$ in Equation (47) for the continuous system will be examined in the following numerical examples. To sum up, rigid body mode 
$\{1,1, \ldots, 1,1\}^{\mathrm{T}}$ and spurious mode $\left\{\psi_{1}\right\}$ satisfy

$$
\begin{aligned}
& {[T]\left\{\begin{array}{c}
1 \\
1 \\
\vdots \\
1 \\
1
\end{array}\right\}=[U]\left\{\begin{array}{c}
0 \\
\vdots \\
0 \\
0 \\
{[T]} \\
\vdots \\
0 \\
0 \\
0
\end{array}\right\}=[U]\left\{\psi_{1}\right\}} \\
& \left\{\begin{array}{c}
0 \\
0
\end{array}\right\}
\end{aligned}
$$

respectively.

\section{THREE REGULARIZATION TECHNIQUES TO DEAL WITH DEGENERATE SCALE PROBLEMS IN BEM}

\subsection{Method of adding a rigid body mode}

Since the $[U]$ matrix is singular in case of the degenerate scale, the modified fundamental solution can be added by a rigid body term $c$,

$$
U^{*}(s, x)=U(s, x)+c
$$

The influence matrix $[U]$ is modified to $\left[U^{*}\right]$, where the component form for the element is

$$
U_{i j}^{*}=U_{i j}+c l_{j}, \quad(i, j=1, \ldots, 2 N)
$$

The zero singular value in $[U]$ moves to a non-zero value for $\left[U^{*}\right]$. To demonstrate the effectiveness, the minimum singular value after the modified fundamental solution will be shown in the numerical examples.

\subsection{Hypersingular formulation}

Instead of using the Equation (16) in the conventional BEM, the second equation of Equation (17) in the dual BEM is used. To demonstrate the idea, the singular value for the $[L]$ matrix will be shown to be non-zero no matter what the expansion ratio is in the following numerical examples. 


\subsection{CHEEF concept}

Since the $[U]$ matrix is singular, the rank is deficient. In order to promote the rank, the independent constraint is required. To resort to the null-field integral equation by collocating the point outside the domain, we have

$$
\langle w\rangle\{t\}=\langle v\rangle\{u\}
$$

where $\langle w\rangle$ and $\langle v\rangle$ are the influence row vectors by collocating the exterior point in the null-field integral equation. By combining Equations (16) with (78), we have

$$
\left[\begin{array}{c}
{[U]_{2 N \times 2 N}} \\
\langle w\rangle_{1 \times 2 N}
\end{array}\right]\{t\}_{2 N \times 1}=\left[\begin{array}{c}
{[T]_{2 N \times 2 N}} \\
\langle v\rangle_{1 \times 2 N}
\end{array}\right]\{u\}_{2 N \times 1}
$$

According to the Equation (79), we can obtain the reasonable solution by using either the least squares method or the SVD technique.

\section{NUMERICAL EXAMPLES}

In this section, three cases including elliptical, square, triangular bars are considered.

\subsection{Elliptical bar}

For the elliptical bar with axes $\alpha m$ and $\beta m(\alpha=3 \beta)$ under torsion, the analytical solution for the conjugate warping function is [36]

$$
u\left(x_{1}, x_{2}\right)=\frac{\alpha^{2}\left(2 \beta^{2}+x_{1}^{2}-x_{2}^{2}\right)+\beta^{2}\left(-x_{1}^{2}+x_{2}^{2}\right)}{2\left(\alpha^{2}+\beta^{2}\right)}, \quad\left(x_{1}, x_{2}\right) \in D
$$

and the boundary flux is

$$
\frac{\partial u}{\partial n}=-\frac{\left(\alpha^{2}-\beta^{2}\right)\left(-\beta^{2} x_{1}^{2}+\alpha^{2} x_{2}^{2}\right)}{\left(\alpha^{2}+\beta^{2}\right) \sqrt{\beta^{4} x_{1}^{2}+\alpha^{4} x_{2}^{2}}}
$$

The torsion rigidity, $T_{\mathrm{r}}$, is

$$
T_{\mathrm{r}}=G \frac{\pi \alpha^{3} \beta^{3}}{\alpha^{2}+\beta^{2}}
$$

The non-trivial boundary mode $\left\{\psi_{1}\right\}$ obtained in Equation (73) in the BEM and the analytical solution $\psi_{1}(\eta)$ using Equation (47) matched well in Figure 6. Good agreement for the numerical data of Equation (70) and the exact solution for the spurious mode is obtained in Figure 6. Table II shows the torsional rigidity obtained by using different approaches. The conventional BEM can work well for the normal case. However, the numerical instability results in a deteriorated solution using BEM when the degenerate scale $(\alpha+\beta=2)$ occurs in the shadow area of Table II. Good agreement was obtained in comparison with the analytical solutions after using the regularization techniques as shown in Table II. 


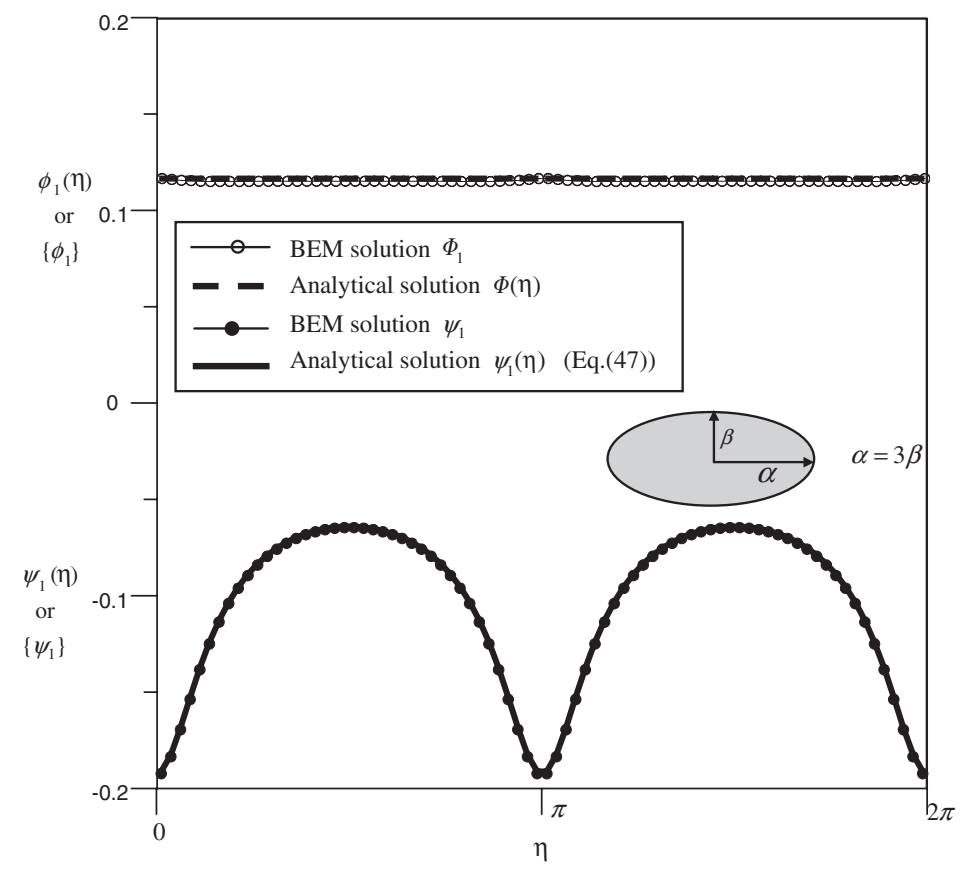

Figure 6. Spurious modes of $\left\{\phi_{1}^{(U)}\right\}$ and $\left\{\psi_{1}^{(U)}\right\}$ in comparison with the analytical solutions for the elliptical bar of the degenerate scale.

By using the conventional BEM, the zero singular value occurs in case of degenerate scale. After adding the rigid body term, $c$, in the fundamental solution, the zero singular value moves to another place by a factor $\mathrm{e}^{-c}$ instead of the original one as shown in Figure 7(a). To investigate how seriously the rank deficiency behaves, we plot the second minimum singular value versus the expansion ratio in Figure $7(\mathrm{~b})$. It indicates that the rank is deficient by one only. This supports us that only one CHEEF point is sufficient. The zero singular value disappears in Figure 7(c) for the $[L]$ matrix in the hypersingular formulation. In order to avoid hypersingularity, the CHEEF method by collocating one point outside the domain can promote the rank as shown in Figure 7(d). Since no zero solution outside the domain can be found in Figure 3(c), the selected CHEEF points are always valid.

\subsection{Square bar}

For the square bar with area $4 a^{2} \mathrm{~m}^{2}$ under torsion, the analytical solution for the conjugate warping function is [36]

$$
u\left(x_{1}, x_{2}\right)=a^{2}+\frac{1}{2}\left(x_{1}^{2}-x_{2}^{2}\right)-\frac{32 a^{2}}{\pi^{3}} \sum_{n=0}^{\infty} \frac{(-1)^{n} \lambda_{n} \cosh \left(\lambda_{n} x_{2}\right) \cos \left(\lambda_{n} x_{1}\right)}{(2 n+1)^{3} \cosh \left(\lambda_{n} a\right)}, \quad\left(x_{1}, x_{2}\right) \in D
$$




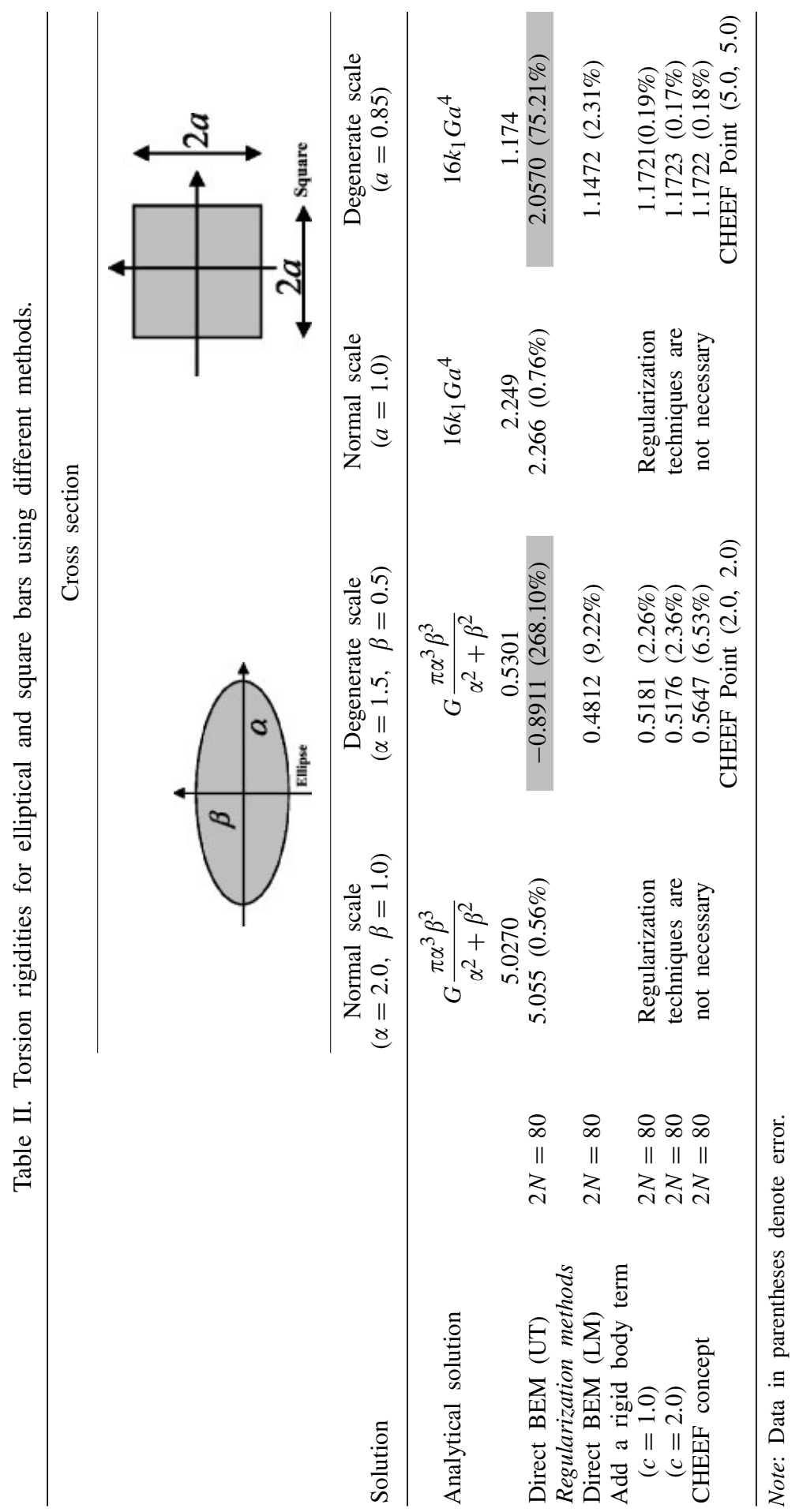

Copyright (c) 2004 John Wiley \& Sons, Ltd. Int. J. Numer. Meth. Engng 2005; 62:233-261 

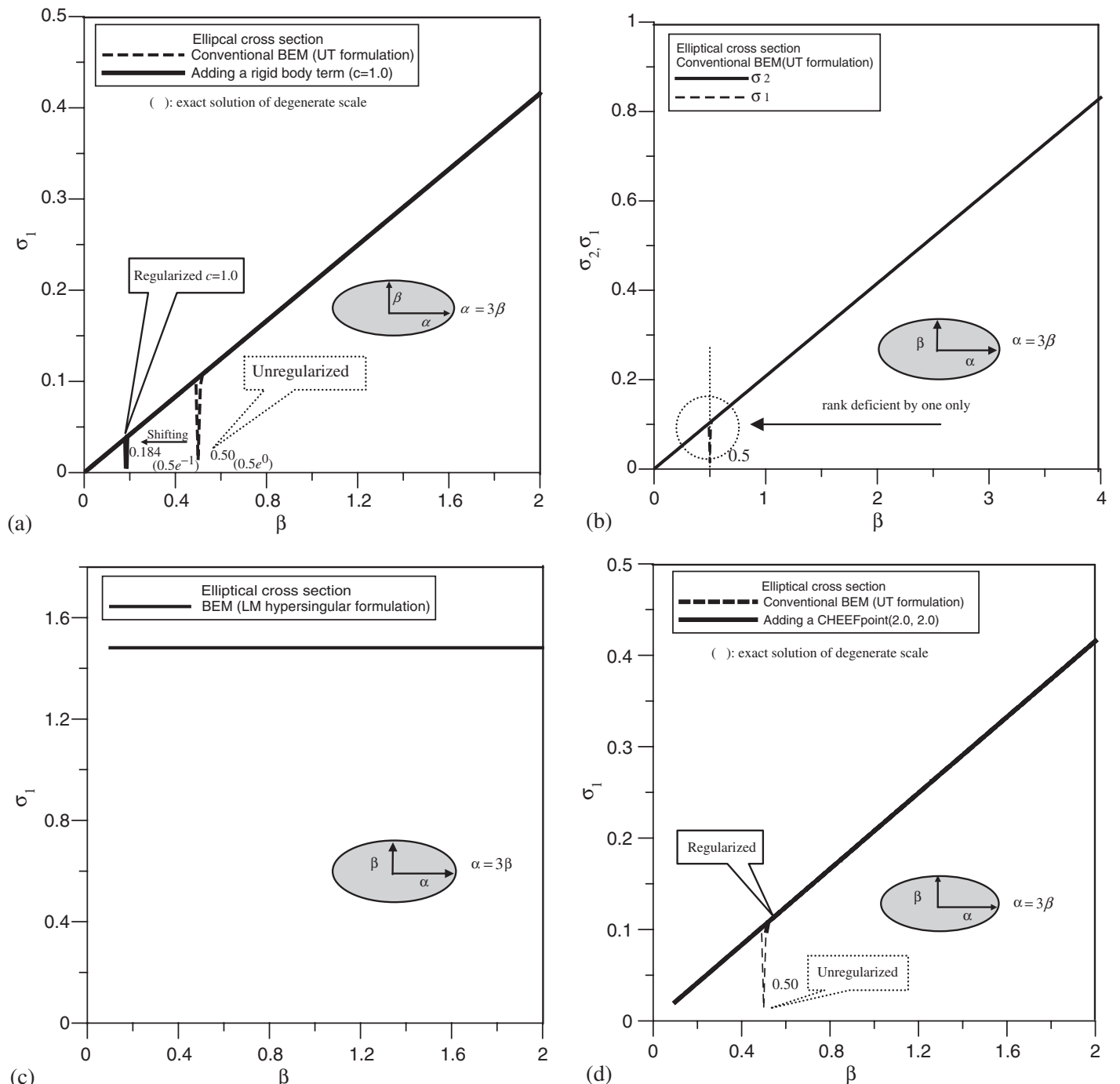

Figure 7. (a) The first minimum singular value versus scale using the conventional BEM (UT formulation) and method of adding a rigid body term; (b) the second minimum singular value versus scale using the conventional BEM (UT formulation); (c) the first minimum singular value versus scale by using the hypersingular equation (LM formulation); and (d) the first minimum singular value versus scale by using the conventional BEM (UT formulation) and CHEEF method.

where

$$
\lambda_{n}=(2 n+1) \frac{\pi}{2 a}, \quad n=0,1,2 \ldots
$$

The boundary flux is

$$
\frac{\partial u}{\partial n}=\frac{\partial u}{\partial x_{1}} n_{x_{1}}+\frac{\partial u}{\partial x_{2}} n_{x_{2}}, \quad\left(x_{1}, x_{2}\right) \in B
$$




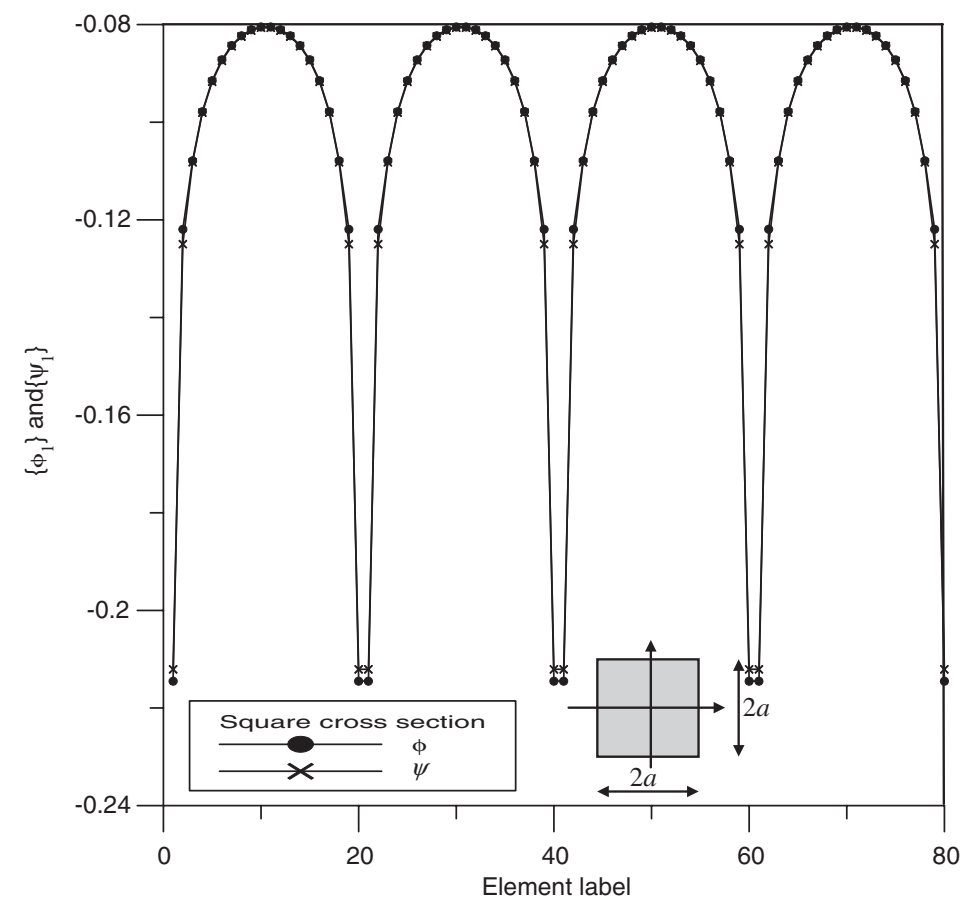

Figure 8. Spurious modes of $\left\{\phi_{1}^{(U)}\right\}$ and $\left\{\psi_{1}^{(U)}\right\}$ for the square bar of the degenerate scale.

where

$$
\begin{aligned}
& \frac{\partial u}{\partial x_{1}}=-x_{1}-\frac{32 a^{2}}{\pi^{3}} \sum_{n=0}^{\infty} \frac{(-1)^{n+1} \lambda_{n} \cosh \left(\lambda_{n} x_{2}\right) \sin \left(\lambda_{n} x_{1}\right)}{(2 n+1)^{3} \cosh \left(\lambda_{n} a\right)} \\
& \frac{\partial u}{\partial x_{2}}=x_{2}-\frac{32 a^{2}}{\pi^{3}} \sum_{n=0}^{\infty} \frac{(-1)^{n} \lambda_{n} \sinh \left(\lambda_{n} x_{2}\right) \cos \left(\lambda_{n} x_{1}\right)}{(2 n+1)^{3} \cosh \left(\lambda_{n} a\right)}
\end{aligned}
$$

and $n_{x_{1}}$ and $n_{x_{2}}$ are the components of the normal vector on the boundary.

The torsional rigidity, $T_{\mathrm{r}}$, of a square bar is

$$
T_{\mathrm{r}}=16 k_{1} G a^{4}
$$

where

$$
k_{1}=\frac{1}{3}\left(1-\frac{192}{\pi^{5}} \sum_{n=0}^{\infty} \frac{\tanh \left(\lambda_{n} a\right)}{(2 n+1)^{5}}\right)
$$

Table II shows the torsional rigidity by using different approaches. In the same way, the conventional BEM (UT formulation) cannot obtain the acceptable results for the case of the degenerate scale as shown in Table II. Figure 8 shows the spurious modes of $\left\{\phi_{1}\right\}$ and $\left\{\psi_{1}\right\}$. 

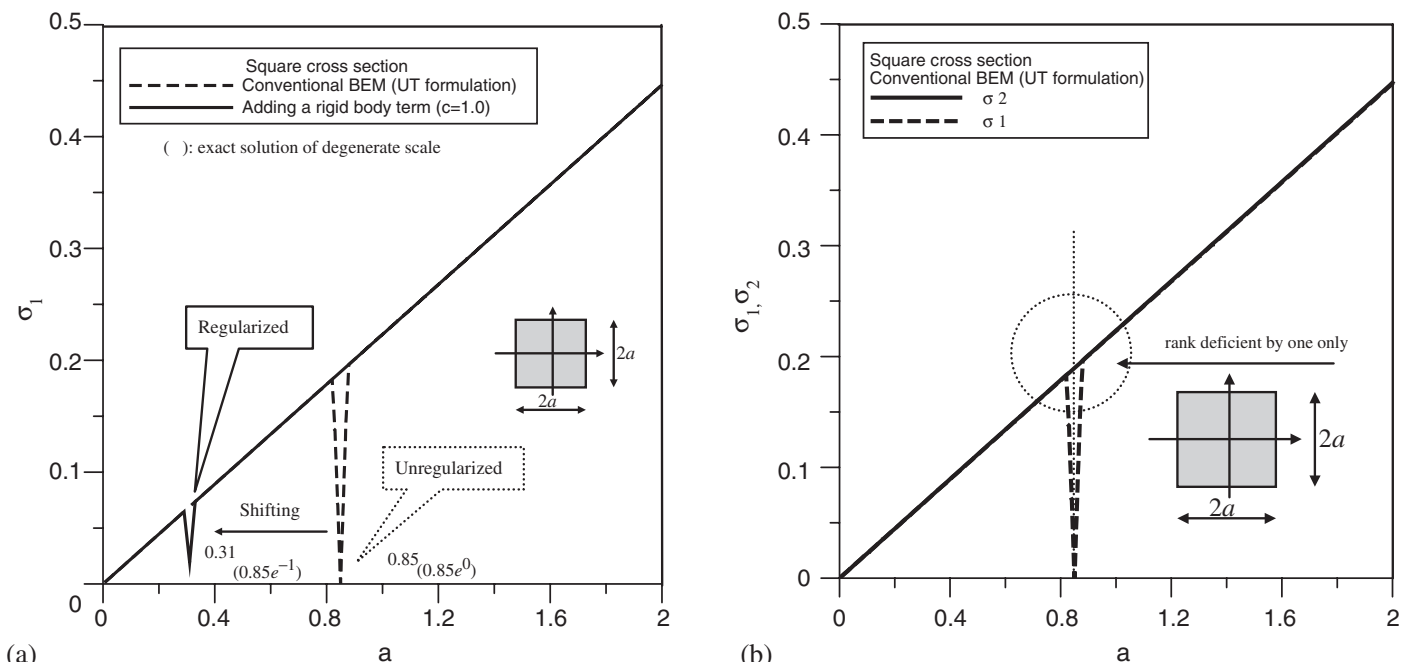

(a)

(b)
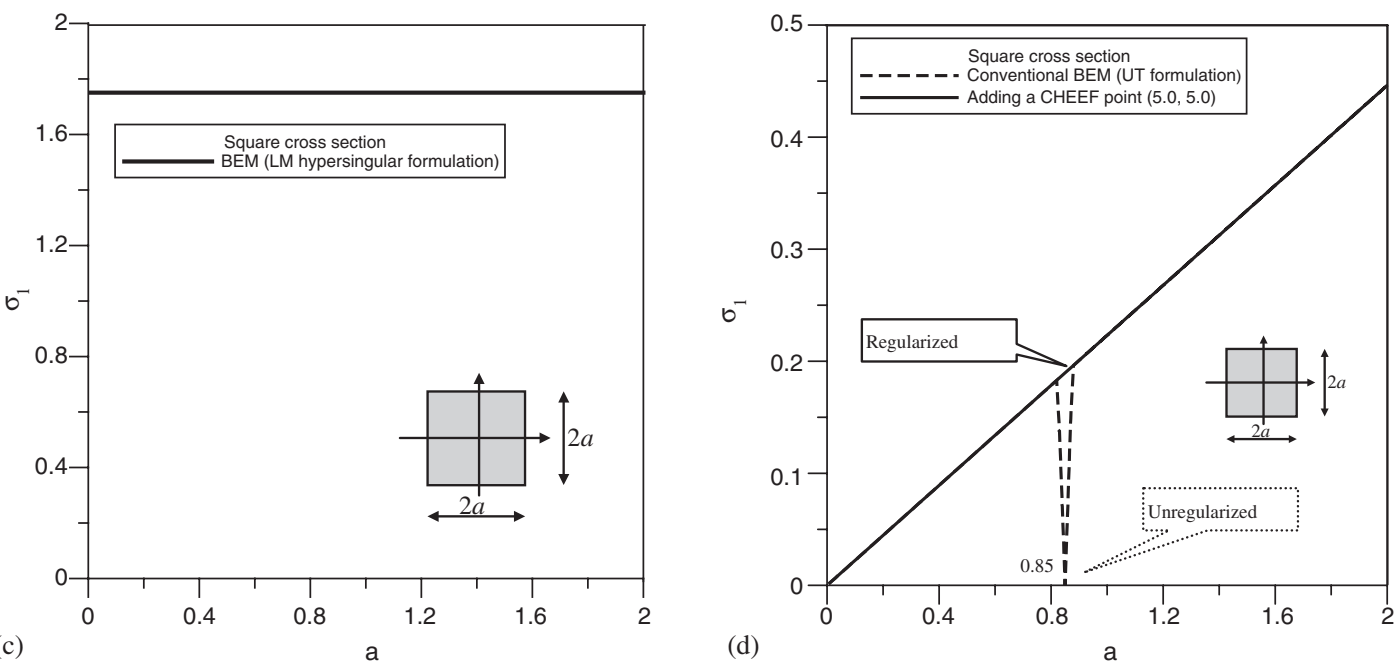

Figure 9. (a) The first minimum singular value versus scale using the conventional BEM (UT formulation) and method of adding a rigid body term; (b) the second minimum singular value versus scale using the conventional BEM (UT formulation); (c) the first minimum singular value versus scale by using the hypersingular equation (LM formulation); and (d) the first minimum singular value versus scale by using the conventional BEM (UT formulation) and CHEEF method.

In this case, no analytical solution can be compared with. By using the conventional BEM, the zero singular value occurs in case of the degenerate scale. After adding the rigid body term in the fundamental solution, the zero singular value moves to another degenerate scale instead of original one as shown in Figure 9(a). To investigate how seriously the rank deficiency behaves, we plot the second minimum singular value versus the expansion ratio in Figure 9(b). 
Table III. Torsion rigidities for a triangular bar using different methods.

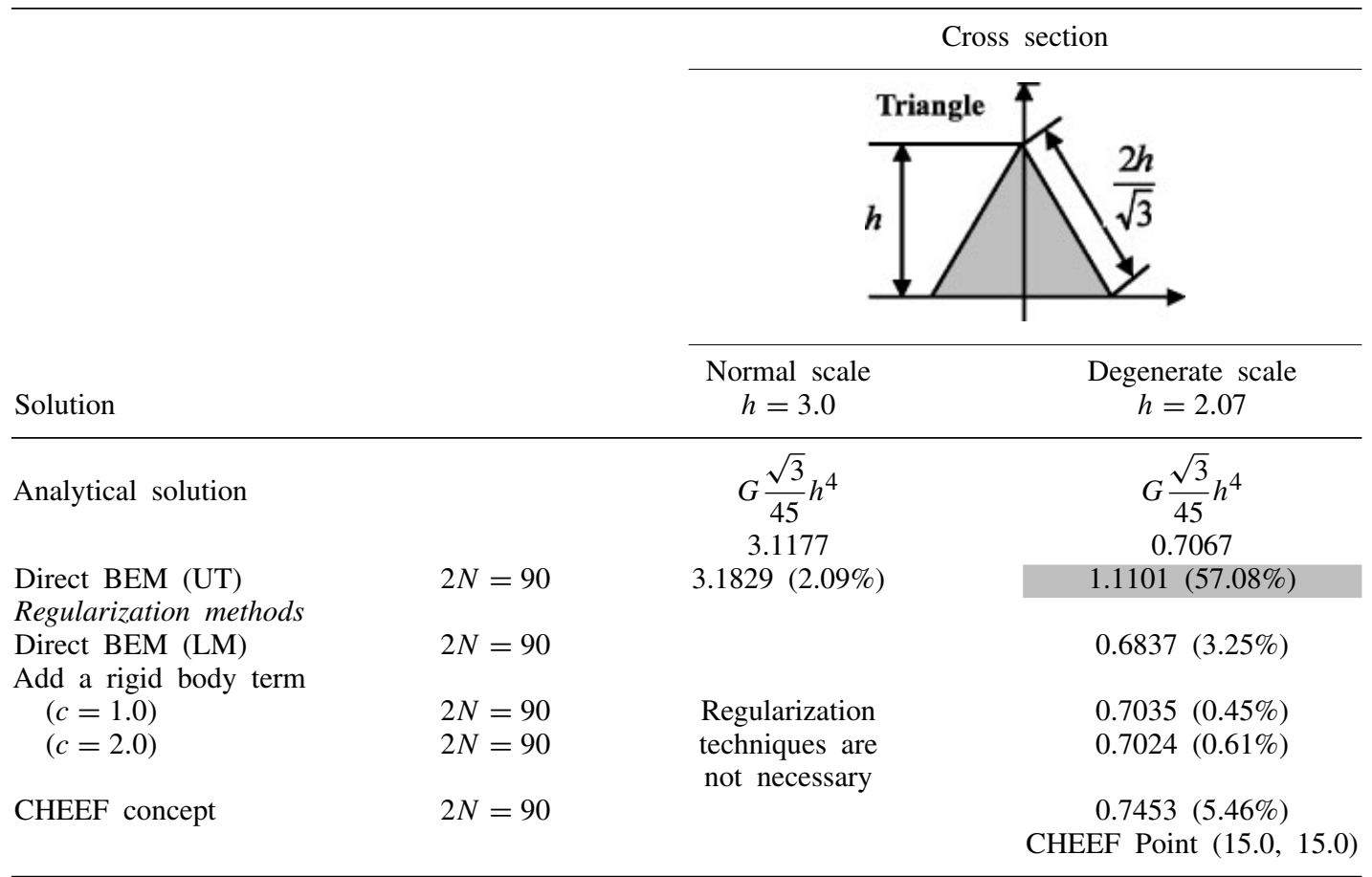

Note: Data in parentheses denote error.

It indicates that rank is deficient by one only. This supports us that only one CHEEF point is required. By employing the hypersingular equation in the dual BEM, it is found that the singular value of $[L]$ matrix for any scale is non-zero as shown in Figure 9(c). In order to avoid hypersingularity, the CHEEF concept by collocating one point outside the domain can promote the rank as shown in Figure $9(\mathrm{~d})$.

\subsection{Triangular bar}

For the equilateral triangular bar with the height $h m$ under torsion, the analytical solution for the conjugate warping function is [36]

$$
u\left(x_{1}, x_{2}\right)=-\frac{1}{2 h}\left(3 x_{2} x_{1}^{2}-x_{2}^{3}+h x_{2}^{2}-h x_{1}^{2}+h^{2} x_{2}\right), \quad\left(x_{1}, x_{2}\right) \in D
$$

and the boundary flux is

$$
\frac{\partial u}{\partial n}=\frac{\partial u}{\partial x_{1}} n_{x_{1}}+\frac{\partial u}{\partial x_{2}} n_{x_{2}}, \quad\left(x_{1}, x_{2}\right) \in B
$$




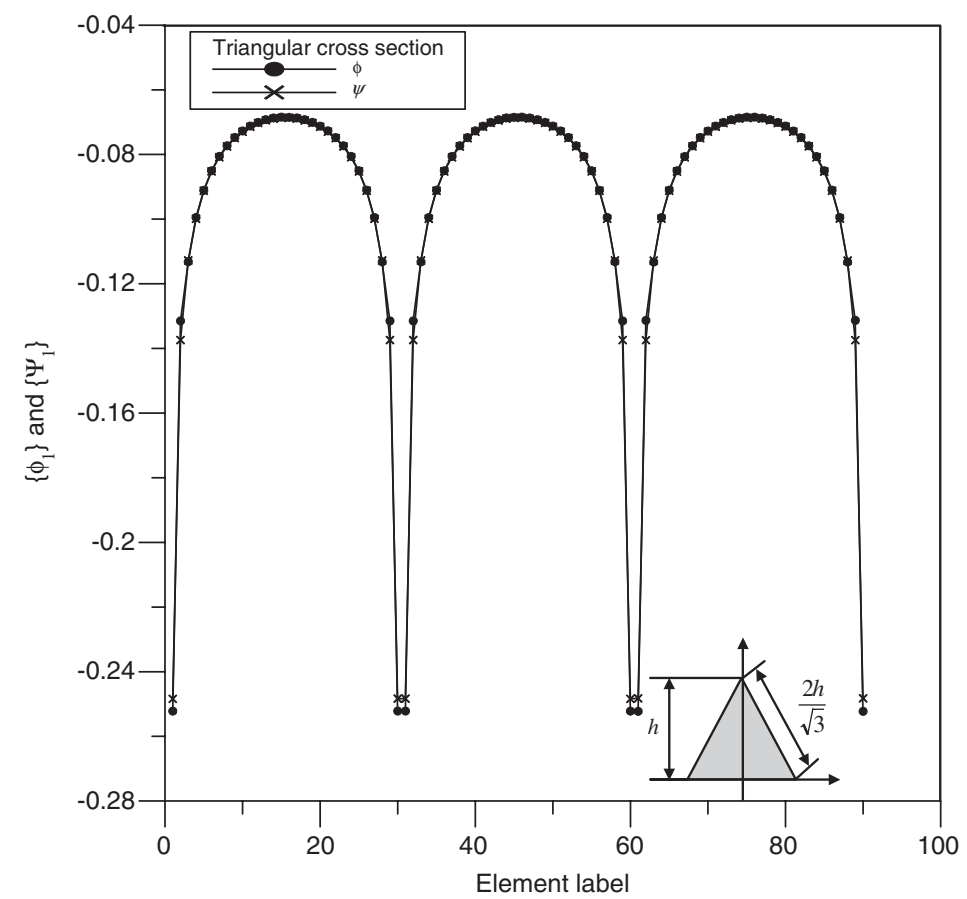

Figure 10. Spurious modes of $\left\{\phi_{1}^{(U)}\right\}$ and $\left\{\psi_{1}^{(U)}\right\}$ for the triangular bar of the degenerate scale.

where

$$
\begin{aligned}
& \frac{\partial u}{\partial x_{1}}=-\frac{1}{2 h}\left(6 x_{2} x_{1}-2 h x_{1}\right) \\
& \frac{\partial u}{\partial x_{2}}=-\frac{1}{2 h}\left(3 x_{1}^{2}-3 x_{2}^{2}+2 h x_{2}+h^{2}\right)
\end{aligned}
$$

The torsion rigidity, $T_{\mathrm{r}}$, is

$$
T_{\mathrm{r}}=G \frac{\sqrt{3}}{45} h^{4}
$$

Table III shows the torsional rigidity by using different approaches. In the same way, the conventional BEM (UT formulation) cannot obtain the acceptable results for the case of degenerate scale as shown in Table III. Figure 10 shows the spurious modes of $\left\{\phi_{1}\right\}$ and $\left\{\psi_{1}\right\}$. In this case, no analytical solution can be compared with. By using the conventional BEM, the zero singular value occurs in case of the degenerate scale. After adding the rigid body term in the fundamental solution, the zero singular value moves to another degenerate scale instead of original one as shown in Figure 11(a). To investigate how seriously the rank deficiency behaves, we plot the second minimum singular value versus the expansion ratio in Figure 11(b). 

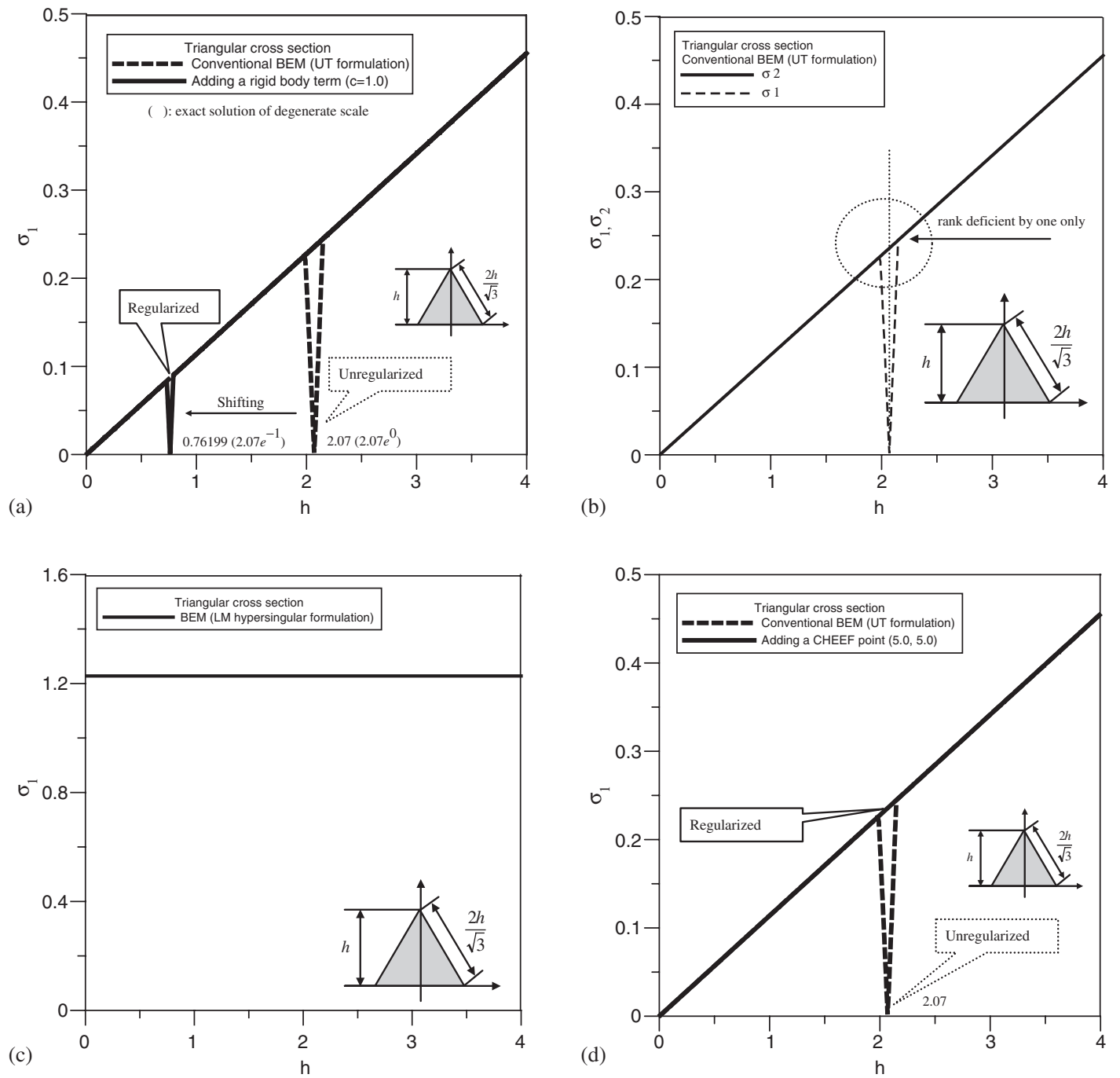

Figure 11. (a) The first minimum singular value versus scale using the conventional BEM (UT formulation) and method of adding a rigid body term; (b) the second minimum singular value versus scale using the conventional BEM (UT formulation); (c) the first minimum singular value versus scale by using the hypersingular equation (LM formulation); and (d) the first minimum singular value versus scale by using the conventional BEM (UT formulation) and CHEEF method.

It indicates that rank is deficient by one only. It is found that the singular value of $[L]$ matrix in the hypersingular equation for any scale is non-zero as shown in Figure 11(c). In order to avoid hypersingularity, the CHEEF method by collocating one point outside the domain can promote the rank as shown in Figure 11(d). 


\section{CONCLUSIONS}

In this paper, the numerical instability for Laplace problems with a degenerate scale by using the conventional BEM was addressed. Torsion bar is chosen as an demonstrative application. Instead of direct searching for the degenerate scale by trial and error, a more efficient technique is proposed to directly determine the singular case since only one normal scale needs to be computed. The degenerate scale for the torsion bar with an elliptical section was derived analytically in the continuous system using the elliptical co-ordinate. For the discrete system, the source of numerical instability is found to be the spurious modes (left and right unitary vectors in SVD with respect to the zero singular value) which were obtained by using the Fredholm alternative theorem and SVD updating document. To deal with the numerical instability due to the degenerate scale, three approaches, method of adding a rigid body mode, hypersingular formulation and CHEEF method, were successfully applied to remove the zero singular value. Good agreement between the BEM results and the analytical solutions were obtained if the regularization techniques are used. Numerical examples, including an elliptical bar, a square bar and triangular bar were demonstrated to check the validity.

\section{ACKNOWLEDGEMENTS}

Financial support from the National Science Council under Grant No. NSC-90-2211-E-019-021 for

National Taiwan Ocean University is gratefully acknowledged. The authors also thank for the discussions on the degenerate scale of the elliptical bar with Prof. S. R. Kuo and Mr J. H. Lin.

\section{REFERENCES}

1. Banerjee PK, Watson JO. Industrial Applications of Boundary Element Methods. Elsevier: New York, 1989.

2. Cruse TA. Recent advances in boundary element analysis method. Computer Methods in Applied Mechanics and Engineering 1997; 62:227-244.

3. Burton AJ, Miller GF. The application of integral equation methods to numerical solutions of some exterior boundary value problems. Proceedings of the Royal Society of London, Series A 1971; 323:201-210.

4. Chen JT, Lin JH, Kuo SR, Chyuan SW. Boundary element analysis for the Helmholtz eigenvalue problems with a multiply connected domain. Proceedings of the Royal Society of London, Series A 2001; 457(2014):2521-2546.

5. Schenck HA. Improved integral formulation for acoustic radiation problems. Journal of the Acoustical Society of America 1976; 44(1):41-58.

6. Chen IL, Chen JT, Liang MT. Analytical study and numerical experiments for radiation and scattering problems using the CHIEF method. Journal of Sound and Vibration 2002; 248(5):809-828.

7. Chen IL, Chen JT, Kuo SR, Liang MT. A new method for true and spurious eigensolutions of arbitrary cavities using the combined Helmholtz exterior integral equation method. Journal of the Acoustical Society of America 2001; 109(3):982-998.

8. Chen JT, Wong FC. Analytical derivations for one-dimensional eigenproblems using dual BEM and MRM. Engineering Analysis with Boundary Elements 1997; 20(1):25-33.

9. Kuo SR, Chen JT, Huang CX. Analytical study and numerical experiments for true and spurious eigensolutions of a circular cavity using the real-part BEM. International Journal for Numerical Methods in Engineering 2000; 48(9):1401-1422.

10. Chen JT, Kuo SR, Chen KH. A nonsingular formulation for the Helmholtz eigenproblems of a circular domain. Journal of the Chinese Institute of Engineering 1999; 22(6):729-740.

11. Chen JT, Hong H-K. Review of dual boundary element methods with emphasis on hypersingular integrals and divergent series. Applied Mechanics Reviews 1999; 52(1):17-33.

12. Lutz ED, Ingraffea AR, Gray LJ. Use of 'simple solutions' for boundary integral methods in elasticity and fracture analysis. International Journal for Numerical Methods in Engineering 1992; 35:1737-1751.

13. Christiansen S. Integral equations without a unique solution can be made useful for solving some plane harmonic problems. Journal of the Institute of Mathematics and its Applications 1975; 16:143-159. 
14. Petrovsky IG. Lectures on Partial Differential Equations. Interscience: New York, 1971.

15. Jaswon MA, Symm GT. Integral Equation Methods in Potential Theory and Electrostatics. Academic Press: New York, 1977.

16. Christiansen S. Derivation and analytical investigation of three direct boundary integral equations for the fundamental biharmonic problem. Journal of Computational and Applied Mathematics 1998; 91:231-247.

17. Christiansen S. Detecting non-uniqueness of solutions to biharmonic integral equations through SVD. Journal of Computational and Applied Mathematics 2001; 134:23-35.

18. He WJ. An equivalent boundary element integral formulation for bending problems of thin plates. Computers and Structures 2000; 74:319-322.

19. He WJ, Ding HJ, Hu HC. Non-equivalence of the conventional boundary integral formulation and its elimination for plane elasticity problems. Computers and Structures 1996; 59(6):1059-1062.

20. He WJ, Ding HJ, Hu HC. Degenerate scales and boundary element analysis of two dimensional potential and elasticity problems. Computers and Structures 1996; 60(1):155-158.

21. He WJ, Ding HJ, Hu HC. A necessary and sufficient boundary element integral formulation for plane elasticity problems. Communications in Numerical Methods in Engineering 1996; 12:413-424.

22. Zhou SJ, Sun SX, Cao ZY. The boundary contour method based on the equivalent boundary integral equation for 2-D linear elasticity. Communications in Numerical Methods in Engineering 1999; 15(11): $811-821$.

23. Tomlinson K, Bradley C, Pullan A. On the choice of a derivative boundary element formulation using Hermite interpolation. International Journal for Numerical Methods in Engineering 1996; 39(3):451-468.

24. Mitra AK, Das S. Nonuniqueness in the integral equations formulation of the biharmonic equation in multiply connected domains. Computer Methods in Applied Mechanics and Engineering 1988; 69:205-214.

25. Chen JT, Lee CF, Chen IL, Lin JH. An alternative method for degenerate scale problems in boundary element method for the two-dimensional Laplace equation. Engineering Analysis with Boundary Elements 2002; 26:559-569.

26. Chen JT, Lin JH, Kuo SR, Chiu YP. Analytical study and numerical experiments for degenerate scale problems in boundary element method using degenerate kernels and circulants. Engineering Analysis with Boundary Elements 2001; 25(9):819-828.

27. Chen JT, Kuo SR, Lin JH. Analytical study and numerical experiments for elasticity plane problem with degenerate scale problems in boundary element method. International Journal for Numerical Methods in Engineering 2002; 54:1669-1681.

28. Kuhn G. BEM in elastostatics and fracture mechanics in 'Finite elements and boundary element techniques from mathematical and engineering point of view'. In International Center for Mechanical Science (ICMS), Courses and Lectures, Stein E, Wendland W (eds). Springer: Berlin, 1988.

29. Constanda C. On non-unique solutions of weakly singular integral equations in plane elasticity. Quarterly Journal of Mechanics and Applied Mathematics 1994; 47:261-268.

30. Constanda C. Integral equations of the first kind in plane elasticity. Quarterly Journal of Mechanics and Applied Mathematics 1995; 53:783-793.

31. Hansen PC, Christiansen S. An SVD analysis of linear algebraic equations derived from first kind integral equations. Journal of Computational and Applied Mathematics 1985; 12,13:341-357.

32. Christiansen S. A review of some integral equations for solving the Saint-Venant torsion problem. Journal of Elasticity 1978; 8(1):1-20.

33. Christiansen S. On two methods for elimination non-unique solutions of an integral equation with logarithmic kernel. Applied Analysis 1982; 13:1-18.

34. Christiansen S. Condition number of matrices derived from two classes of integral equations. Mathematical Methods in the Applied Sciences 1981; 3:364-392.

35. Chen JT, Chen KH, Yeih W, Shieh NC. Dual boundary element analysis for cracked bars under torsion. Engineering Computations 1998; 15(6):732-749.

36. Reismann H, Pawlik PS. Elasticity: Theory and Applications. Wiley: New York, 1980.

37. Chen JT, Hong H-K. Boundary Element Method (2nd edn). New World Press: Taipei, Taiwan, 1992 (in Chinese).

38. Lin JH. Study on the degenerate scale and the multiply-connected acoustic cavity. Master Thesis. Department of Harbor and River Engineering, National Taiwan Ocean University, Keelung, Taiwan, 2000.

39. Davis HT, Thomson KT. Linear Algebra and Linear Operators in Engineering with Applications in Mathematica. Academic Press: New York, 2000. 